\title{
On Unique Continuation for Navier-Stokes Equations
}

\author{
Zhiwen Duan, Shuxia Han, and Peipei Sun \\ Department of Mathematics, Huazhong University of Science and Technology, Wuhan 430074, China \\ Correspondence should be addressed to Shuxia Han; 1998259@hust.edu.cn
}

Received 4 June 2014; Accepted 25 August 2014

Academic Editor: BoQing Dong

Copyright (C) 2015 Zhiwen Duan et al. This is an open access article distributed under the Creative Commons Attribution License, which permits unrestricted use, distribution, and reproduction in any medium, provided the original work is properly cited.

\begin{abstract}
We study the unique continuation properties of solutions of the Navier-Stokes equations. We take advantage of rotation transformation of the Navier-Stokes equations to prove the "logarithmic convexity" of certain quantities, which measure the suitable Gaussian decay at infinity to obtain the Gaussian decay weighted estimates, as well as Carleman inequality. As a consequence we obtain sufficient conditions on the behavior of the solution at two different times $t_{0}=0$ and $t_{1}=1$ which guarantee the "global" unique continuation of solutions for the Navier-Stokes equations.
\end{abstract}

\section{Introduction}

In this paper, we study the unique continuity of the NavierStokes equations:

$$
\begin{gathered}
u_{t}-\Delta u+u \cdot \nabla u+\nabla p=0 \quad \text { in } \mathbf{R}^{n} \times(0,1), \\
\nabla \cdot u=0 \quad \text { in } \mathbf{R}^{n} \times(0,1) .
\end{gathered}
$$

For (1), the existence of the Leray solutions [1] can be found in [2-4] (see also [5-9] for the existence of the general solutions). The regularity of (1) $(n \geq 3)$ is an open problem, but some results of the partial regularity can refer to [10-12].

Due to the fact that our consequence needs some asymptotic behavior of the solutions to (1) as conditions, we first mention some the space-time asymptotic behavior of the solutions. In [13], Amrouch et al. studied the space-time asymptotic behavior of the solutions, and their derivatives, to (1) in dimension $2 \leq n \leq 5$, and obtained that the strong solutions to (1) which decay in $L^{2}$ at the rate of $\|u(t)\|_{2} \leq$ $C(t+1)^{-\mu}$ have the following point-wise space-time decay, for $0 \leq k \leq n / 2$,

$$
\left|D^{\alpha} u(x, t)\right| \leq C_{k, m} \frac{1}{(t+1)^{\rho_{0}}\left(1+|x|^{2}\right)^{k / 2}},
$$

with $\rho_{0}=(1-2 k / n)(m / 2+\mu+n / 4),|\alpha|=m$, and $\mu>$ $(n / 4)$. The $L^{2}$ decay for solutions of (1) was studied in [1417]. On other hand, the backward uniqueness for parabolic equations will be used in our paper. In [18], Escauriaza et al. proved a backward uniqueness result for the heat operator with variable lower order terms, which implies the full regularity of $L_{3, \infty}$-solutions of the three-dimensional NavierStokes equations. Some backward results of the Navier-Stokes equations can refer to $[19,20]$.

The unique continuation is best understood for second order elliptic operators, in which the powerful technique socalled Carleman weighted estimate played a central role (see [21, Chapter 17] and [22]). In [21, Chapter 28], the Calderón uniqueness theorems for some general linear partial differential operators were obtained by Carleman estimates. The proofs in Chapter 28 of [21] relied on factorization in first order pseudodifferential operators. A careful study of these factors led to more general forms of the Calderón uniqueness theorem. In [23], Saut and Scheurer proved a unique continuation theorem when $L$ was a second order parabolic equation in the first section. Their proof is simple and based on the derivation of a Carleman estimate which is reminiscent of the classical Carleman estimates for second order elliptic operators. This Carleman inequality allows the weakening of the smoothness assumptions on the principal operator $L$. And they extended also these results to some mixed parabolic-elliptic systems and some higher order parabolic equations. For the parabolic equations [24], the stokes equations [25], and the Navier-Stokes equations $[26,27]$, the similar "local (space)" uniqueness results were 
obtained. In [28], interpolation arguments and Sobolev imbedding theorem led to an $L^{p}(p>2)$ Carleman estimate therefore to a unique continuation theorem. In [29-32], the "global" unique continuation for the Schrödinger equations was discussed.

For the stationary Navier-Stokes equations:

$$
\begin{gathered}
-\Delta u+u \cdot \nabla u+\nabla p=0 \quad \text { in } \Omega, \\
\nabla \cdot u=0 \quad \text { in } \Omega,
\end{gathered}
$$

in three dimensions, Finn [33] showed that $\left.u\right|_{\partial B_{R}}=0$ and $u=o\left(|x|^{-1}\right)$; then, $u$ is trivial $\left(\Omega=\mathbf{R}^{3} \backslash B_{R}(0)\right)$. Later Dyer and Edmunds [34] proved that if $u$ is $C^{2}$ bounded, and $u=O\left(\exp \left(-\exp \left(\alpha|x|^{3}\right)\right)\right)$ for all $\alpha>0$, then $u$ is trivial (see also [35]). In [36], Lin et al. showed that, for $n=2,3$, if $u$ is bounded in $\Omega$, then any nontrivial of (3) cannot decay faster than certain double exponential at infinity (see Corollary 1.6 [36]). In [37], they improved on this result in [36] and studied the asymptotic behavior of solutions of the stationary NavierStokes equations in an exterior domain, through assuming $u \in\left(H_{\mathrm{loc}}^{1}(\Omega)\right)^{n},\|u\|_{L^{\infty}(\Omega)} \leq \lambda(n=2)$, or $\|u\|_{L^{\infty}(\Omega)}+$ $\|\nabla u\|_{L^{\infty}(\Omega)} \leq \lambda(n \geq 3)$ and choosing the appropriate Carleman estimates based on Lemma 2.4 in [38], combining interior estimates, showed that any nontrivial solution obeyed a minimal decaying rate $\exp \left(-C t^{2} \log t\right)$ at infinity. Our goal is to obtain sufficient conditions on the behavior of the solution $u$ at two different times $t_{0}=0$ and $t_{1}=1$ which guarantee that the solution of (1) is trivial.

For the heat equation, applying Hardy's uncertainty principle [39] to $e^{\Delta} f, e^{|x|^{2} / \delta^{2}} e^{\Delta} f$ and $e^{4|\xi|^{2} / 2^{2}} \widehat{e^{\Delta} f}=\widehat{f}$ would be in $L^{2}\left(\mathbf{R}^{n}\right)$, and $2 \delta \leq 4$ implies $e^{\Delta} f \equiv 0$. Then, backward uniqueness arguments [40] show that $f \equiv 0$. Moreover, due to the result in [31]: If $f(x)=O\left(e^{-|x|^{2} / \beta^{2}}\right), \widehat{f}(\xi)=O\left(e^{-|\xi|^{2} / \alpha^{2}}\right)$, and $\alpha \beta<4$, then $f \equiv 0$. This result can be rewritten in terms of the free solution of the Schrödinger equation:

$$
\begin{gathered}
i \partial_{t} u+\Delta u=0, \quad u(0)=f, \quad(x, t) \in \mathbf{R}^{n} \times(0,+\infty), \\
u(x, t)=(2 \pi i t)^{-n / 2} e^{i|x|^{2} /(4 t)} \widehat{e^{i||^{2} /(4 t)}} f\left(\frac{x}{2 t}\right) .
\end{gathered}
$$

That means if $u(x, 0)=O\left(e^{-|x|^{2} / \beta^{2}}\right), u(x, T)=O\left(e^{-|\xi|^{2} / \alpha^{2}}\right)$, then when $\alpha \beta<4 T, u \equiv 0$. By applying "logarithmically convex" and Carleman estimates of equations after the Appel transformation, Escauriaza et al. [31] showed the following.

(1) If $u \in C\left([0,1], L^{2}\left(\mathbf{R}^{n}\right)\right)$ and satisfies

$$
\partial_{t} u=i(\Delta u+V(x, t) u), \quad(x, t) \in \mathbf{R}^{n} \times[0,1],
$$

where $\alpha, \beta$ are positive, $\alpha \beta<2$, and $\left\|e^{|x|^{2} / \beta^{2}} u(0)\right\|_{L^{2}\left(\mathbf{R}^{n}\right)}$, $\left\|e^{|x|^{2} / \alpha^{2}} u(1)\right\|_{L^{2}\left(\mathbf{R}^{n}\right)}$ are finite,

$$
\lim _{R \rightarrow+\infty}\|V\|_{L^{1}\left([0,1], L^{\infty}\left(\mathbf{R}^{n} \backslash B_{R}\right)\right)}=0,
$$

then $u \equiv 0$.
(2) If $u \in L^{\infty}\left([0,1], L^{2}\left(\mathbf{R}^{n}\right)\right) \bigcap L^{2}\left([0, T], H^{1}\left(\mathbf{R}^{n}\right)\right)$ satisfies

$$
\begin{gathered}
u_{t}=\Delta u+V(x, t) u, \quad(x, t) \in \mathbf{R}^{n} \times[0,1], \\
u(0)=f, \quad x \in \mathbf{R}^{n},
\end{gathered}
$$

where $V(x, t)$ is bounded in $\mathbf{R}^{n} \times[0,1]$ and $\delta<1, f$, $e^{|x|^{2} / \delta^{2}} u(1)$ are in $L^{2}\left(\mathbf{R}^{n}\right)$, then $u \equiv 0$.

Based on these results above, it is natural to expect that Hardy's uncertain principle holds on Navier-Stokes equations (1). In this paper, our aim is to prove the the following unique continuation theorem of Navier-Stokes equations (1).

Theorem 1. If $\operatorname{curl} u(x, t) \in\left(C^{2}\left(\mathbf{R}^{n} \times[0,1]\right)\right)^{n \times n}$ satisfies (1) and there are constants $C_{0}, C_{1}, C_{2}$ which satisfy the following inequalities:

$$
\begin{aligned}
& \|u\|_{L^{\infty}\left(\mathbf{R}^{n} \times[0,1]\right)},\|\nabla u\|_{L^{\infty}\left(\mathbf{R}^{n} \times[0,1]\right)},\|\Delta u\|_{L^{\infty}\left(\mathbf{R}^{n} \times[0,1]\right)} \leq C_{0}, \\
& \left\|\partial_{t} u \cdot x\right\|_{L^{\infty}\left(\mathbf{R}^{n} \times[0,1]\right)},\|u \cdot x\|_{L^{\infty}\left(\mathbf{R}^{n} \times[0,1]\right)},\|\nabla u \cdot x\|_{L^{\infty}\left(\mathbf{R}^{n} \times[0,1]\right)} \\
& \leq C_{1}, \\
& \|(x \cdot \nabla) u \cdot x\|_{L^{\infty}\left(\mathbf{R}^{n} \times[0,1]\right)} \leq C_{2} .
\end{aligned}
$$

We also assume that $e^{\gamma|x|^{2}} \operatorname{curl} u(0)$ and $e^{\gamma|x|^{2}} \operatorname{curl} u(1)$ are in $L^{2}\left(\mathbf{R}^{n}\right)$. Then $u \equiv 0$ for $(x, t) \in \mathbf{R}^{n} \times[0,1]$.

Our arrangement is as follows. In Section 2, we introduce variable transformation of the curl; thus, we can reduce the information of the tension item and simplify the equations. Hence we get the equations of the tensor $q=$ curl $u$. In Section 3, using the transformation of weighted function and constructing "logarithmic convexity" of the solutions of the equations about $q$, we get the Gaussian weight $L^{2}$ estimates of $q$. But in this Gaussian weight $L^{2}$ estimates, we need to justify the validity of the arguments in Proposition 5. Due to the fact that the equations of $q$ include the term $(A \cdot \nabla) q$, we cannot use the cut-off method as in [31] to justify the validity. We thus first give a Gaussian weight $L^{2}$ preestimates (see Proposition 2). In Section 4, we prove a Carleman estimate about $q$. By the Carleman estimate above, we mainly stress the unique continuation for the equations about $q$ in $\mathbf{R}^{n} \times[0,1]$. This means we accordingly get the unique continuation for the equation about $q$. According with the conditions that have been given, we lastly analyze the unique continuation for the given Navier-Stokes equations about $u$ in $\mathbf{R}^{n} \times[0,1]$.

We give the notations in this paper.

Let $f(x, t)=\left(f_{i j}(x, t)\right), g(x, t)=\left(g_{i j}(x, t)\right)$ be 2 -order tensor, and define

$$
(f, g)=\int_{\mathbf{R}^{n}} f_{i j}(\cdot, t) \cdot g_{i j}(\cdot, t) d x
$$

$$
\|f\|^{2}=\|f\|_{L^{2}\left(\mathbf{R}^{n}\right)}=(f, f)=\sum\left\|f_{i j}\right\|^{2}
$$




$$
\begin{gathered}
\|f(x, t)\|_{L^{2}\left(\mathbf{R}^{n} \times[0,1]\right)}=\left(\int_{0}^{1}\|f(\cdot, t)\|^{2} d t\right)^{1 / 2}, \\
\|f\|_{\infty}=\sum\left\|f_{i j}\right\|_{L^{\infty}\left(\mathbf{R}^{n} \times[0,1]\right)}, \\
\|f(x, t)\|_{L^{\infty}\left([0,1], L^{2}\left(\mathbf{R}^{n}\right)\right)}=\sup _{0 \leq t \leq 1}\|f\| .
\end{gathered}
$$

$\mathcal{S}, \mathscr{A}$ are the symmetric and skew-symmetric operators, $\delta_{t}$ shows the partial derivative on $t$ about coefficients of the operator $\mathcal{S}$, and commutator $[\mathcal{S}, \mathscr{A}]=\mathcal{S} \mathscr{A}-\mathscr{A} \mathcal{S}$.

$C_{0}^{\infty}$ means $C^{\infty}$ with compact support.

\section{Reduced System}

In order to simplify the equations, we introduce $q$, which is the curl of the solutions of (1):

$$
q_{i j}=(\operatorname{curl} u)_{i j}=\frac{1}{\sqrt{2}}\left(\partial_{i} u_{j}-\partial_{j} u_{i}\right), \quad 1 \leq i, j \leq n .
$$

Thus we transform (1) into equations about $q$.

In detail, we also introduce

$$
\left(\operatorname{curl}^{T} q\right)_{i}=\frac{1}{\sqrt{2}} \sum_{j=1}^{n} \partial_{j}\left(q_{i j}-q_{j i}\right), \quad(1 \leq i \leq n),
$$

where $\operatorname{curl}^{T}$ denotes the transpose of curl. Then it is easy to prove

$$
\Delta u=\nabla(\nabla \cdot u)-\operatorname{curl}^{T} \operatorname{curl} u .
$$

In fact,

$$
\begin{aligned}
& \left(\operatorname{curl}^{T} \operatorname{curl} u\right)_{i} \\
& =\frac{1}{\sqrt{2}} \sum_{j=1}^{n} \partial_{j}\left(\operatorname{curl} u_{i j}-\operatorname{curl} u_{j i}\right) \\
& =\frac{1}{\sqrt{2}} \sum_{j=1}^{n} \partial_{j}\left[\frac{1}{\sqrt{2}}\left(\partial_{i} u_{j}-\partial_{j} u_{i}\right)-\frac{1}{\sqrt{2}}\left(\partial_{j} u_{i}-\partial_{i} u_{j}\right)\right] \\
& =\sum_{j=1}^{n} \partial_{j} \partial_{i} u_{j}-\Delta u_{i} \\
& =[\nabla(\nabla \cdot u)]_{i}-\Delta u_{i} .
\end{aligned}
$$

Noticing that $\nabla \cdot u=0,(12)$ becomes $\Delta u+\operatorname{curl}^{T} q=0$. Moreover,

$$
u \cdot \nabla u=\nabla\left(\frac{1}{2}|u|^{2}\right)-\sqrt{2}(\operatorname{curl} u) u .
$$

Applying curl on the equations $u_{t}-\Delta u+u \cdot \nabla u+\nabla p=0$, we have

$$
q_{t}-\Delta q+Q(q) u+q(\nabla u)^{T}-(\nabla u) q^{T}=0
$$

where $(Q(q) u)_{i j}=\sum_{k=1}^{n}\left(\partial_{j} q_{i k}-\partial_{i} q_{j k}\right) u_{k}=[(u \cdot \nabla) q]_{i j}$. In fact, by the definitions of $q$ and $Q(q)$, we get

$$
\begin{gathered}
{\left[q(\nabla u)^{T}-(\nabla u) q^{T}\right]_{i j}=\frac{1}{\sqrt{2}} \sum_{k=1}^{n}\left[\partial_{i} u_{k} \partial_{k} u_{j}-\partial_{j} u_{k} \partial_{k} u_{i}\right]} \\
{[\operatorname{curl}(u \cdot \nabla u)]_{i j}} \\
=\frac{1}{\sqrt{2}}\left[\partial_{i} \sum_{k=1}^{n} u_{k} \partial_{k} u_{j}-\partial_{j} \sum_{k=1}^{n} u_{k} \partial_{k} u_{i}\right] \\
=\frac{1}{\sqrt{2}}\left[\sum_{k=1}^{n}\left(\partial_{i} u_{k} \partial_{k} u_{j}-\partial_{j} u_{k} \partial_{k} u_{i}\right)\right. \\
\left.+\sum_{k=1}^{n}\left(u_{k} \partial_{i} \partial_{k} u_{j}-u_{k} \partial_{j} \partial_{k} u_{i}\right)\right] \\
=\left[q(\nabla u)^{T}-(\nabla u) q^{T}+(u \cdot \nabla) q\right]_{i j}
\end{gathered}
$$

Therefore

$$
\begin{aligned}
\operatorname{curl}(u \cdot \nabla u) & =Q(q) u+q(\nabla u)^{T}-(\nabla u) q^{T}, \\
(\operatorname{curl}(\Delta u))_{i j}= & \left(\operatorname{curl}\left(-\operatorname{curl}^{T} q\right)\right)_{i j} \\
= & -\frac{1}{\sqrt{2}}\left(\partial_{i}\left(\operatorname{curl}^{T} q_{j}\right)-\partial_{j}\left(\operatorname{curl}^{T} q_{i}\right)\right) \\
= & -\frac{1}{\sqrt{2}}\left(\partial_{i}\left(\frac{1}{\sqrt{2}} \sum_{k=1}^{n} \partial_{k}\left(q_{j k}-q_{k j}\right)\right)\right) \\
& -\partial_{j}\left(\frac{1}{\sqrt{2}} \sum_{k=1}^{n} \partial_{k}\left(q_{i k}-q_{k i}\right)\right) \\
= & -\sum_{k=1}^{n}\left(\partial_{i k} q_{j k}-\partial_{j k} q_{i k}\right) \\
= & -(\Delta q)_{j i}=(\Delta q)_{i j} .
\end{aligned}
$$

By $\operatorname{curl}(\nabla p)=0$, we easily get (15).

For $n=2$, due to $\nabla \cdot u=0$, it is easy to see that $q(\nabla u)^{T}-$ $(\nabla u) q^{T}=0$. Hence, for $n=2$, we only need to consider the system:

$$
q_{t}-\Delta q+Q(q) u=0, \quad(x, t) \in \mathbf{R}^{2} \times[0,1],
$$

for $n \geq 3$, we only need to consider the equations:

$$
\begin{array}{r}
q_{t}-\Delta q+(Q(q) u)+q(\nabla u)^{T}-(\nabla u) q^{T}=0, \\
(x, t) \in \mathbf{R}^{n} \times[0,1] .
\end{array}
$$

In order to get the unique continuation of (1), putting together (18) and (19), it suffices to consider

$$
q_{t}-\Delta q+(A \cdot \nabla) q+B q=0, \quad(x, t) \in \mathbf{R}^{n} \times[0,1],
$$

where $A=u, B q=q(\nabla u)^{T}-(\nabla u) q^{T}$. 


\section{Gaussian Weighted Estimates}

In this section, we consider Gaussian weighted estimates of $q$ and $\nabla q$ in (20); that is,

$$
q_{t}-\Delta q+(A \cdot \nabla) q+B q=0
$$

Proposition 2. If $q \in\left(L^{\infty}\left([0,1], L^{2}\left(\mathbf{R}^{n}\right)\right)\right)^{n \times n} \bigcap\left(L^{2}([0,1]\right.$, $\left.\left.H^{2}\left(\mathbf{R}^{n}\right)\right)\right)^{n \times n}$ satisfies

$$
\partial_{t} q-\Delta q+A \cdot \nabla q+B q=F
$$

$\|A\|_{L^{\infty}\left(\mathbf{R}^{n} \times[0,1]\right)} \leq C_{0}<2 \gamma$, and $\|B\|_{L^{\infty}\left(\mathbf{R}^{n} \times[0,1]\right)} \leq$ $2\|\nabla u\|_{L^{\infty}\left(\mathbf{R}^{n} \times[0,1]\right)} \leq 2 C_{0}$, then

$$
\begin{aligned}
\int e^{\gamma|x|^{2}} & |q(x, t)|^{2} d x \\
\leq & 14^{n / 2} \cdot \int e^{14 \gamma|x|^{2}}|q(x, 0)|^{2} d x \\
& +14^{n / 2} e^{(14 \gamma)^{-1}\left(3 C_{0}^{2}+4 C_{0}+1\right) / 2}(14 \gamma)^{-1} \\
& \cdot \int_{0}^{1} \int \frac{e^{14 \gamma|x|^{2}}|F|^{2}}{2} d x d t,
\end{aligned}
$$

where $\gamma>0$.

Proof. Let $\gamma>0, \bar{q}(\bar{x}, \bar{t})=q\left(\gamma^{-1 / 2} \bar{x}, \gamma^{-1} \bar{t}\right)$; then,

$$
\partial_{\bar{t}} \bar{q}-\Delta \bar{q}+\gamma^{-1 / 2} A \cdot \nabla \bar{q}+\gamma^{-1} B \bar{q}=\gamma^{-1} F .
$$

Denote by $\beta=\left(\beta_{1}, \beta_{2}, \ldots, \beta_{n}\right)$. Without loss of generality, assume that $\beta_{k} \geq 0 \quad(k=1,2, \ldots, n)$. Let $\phi_{N} \in C^{\infty}(\mathbf{R})$ such that

$$
\begin{gathered}
\phi_{N}(s)=1, \quad s \leq N, \\
\phi_{N}(s)=0, \quad s \geq 10 N,
\end{gathered}
$$

with

$$
0 \leq \phi_{N} \leq 1, \quad\left|\phi_{N}^{(j)}(s)\right| \leq \frac{c_{j}}{N^{j}}, \quad j=1,2, \ldots
$$

Let $\vartheta_{k, N}(s)=\beta_{k} \int_{0}^{s} \phi_{N}^{2}(r) d r$, so that $\vartheta_{k, N}(s) \in C^{\infty}(\mathbf{R})$ nondecreasing with

$$
\begin{gathered}
\vartheta_{k, N}(s)=\beta_{k} s, \quad s<N, \\
\vartheta_{k, N}(s)=c_{N} \beta_{k}, \quad s>10 N, \\
\vartheta_{k, N}^{\prime}(s)=\beta_{k} \phi_{N}^{2}(s) \leq \beta_{k}, \\
\left|\vartheta_{k, N}^{(j)}(s)\right| \leq \frac{\beta_{k} c_{j}}{N^{j-1}}, \quad j=1,2, \ldots
\end{gathered}
$$

Let $\psi_{k, N}(s)=e^{2 \vartheta_{k, N}(s)}$ so that $\psi_{k, N}(s) \leq e^{2 \beta s}$ and $\psi_{k, N}(s) \rightarrow$ $e^{2 \beta_{k} s}$ as $N \rightarrow \infty$.

Taking $\psi_{N}(\bar{x})=\psi_{1, N}\left(\bar{x}_{1}\right) \psi_{2, N}\left(\bar{x}_{2}\right) \ldots \psi_{n, N}\left(\bar{x}_{n}\right)$, and

$$
v_{N}(\bar{x}, \bar{t})=\psi_{N}(\bar{x}) \bar{q}(\bar{x}, \bar{t}) \text {. }
$$

Using that

$$
\begin{gathered}
\psi_{N} \bar{q}=v_{N}, \\
\psi_{N} \partial_{\bar{t}} \bar{q}=\partial_{\bar{t}} v_{N}, \\
\psi_{N} \partial_{\bar{x}_{k}} \bar{q}=\partial_{\bar{x}_{k}} v_{N}-2 \beta_{k} \phi_{N}^{2}\left(\bar{x}_{k}\right) v_{N}, \\
\psi_{N} \partial_{\bar{x}_{k}}^{2} \bar{q}=\partial_{\bar{x}_{k}}^{2} v_{N}-4 \beta_{k} \phi_{N}^{2}\left(\bar{x}_{k}\right) \partial_{\bar{x}_{k}} v_{N} \\
-\left(4 \beta_{k} \phi_{N}\left(\bar{x}_{k}\right) \phi_{N}^{\prime}\left(\bar{x}_{k}\right)-4 \beta_{k}^{2} \phi_{N}^{4}\left(\bar{x}_{k}\right)\right) v_{N} .
\end{gathered}
$$

So, one gets the equations of $v_{N}$,

$$
\begin{aligned}
\partial_{\bar{t}} v_{N}= & \Delta v_{N}-\gamma^{-1 / 2} A \cdot \nabla v_{N}-\gamma^{-1} B\left(v_{N}\right) \\
& -4 \sum_{k=1}^{n} \beta_{k} \phi_{N}^{2}\left(\bar{x}_{k}\right) \partial_{\bar{x}_{k}} v_{N}-4 \sum_{k=1}^{n} \beta_{k} \phi_{N}\left(\bar{x}_{k}\right) \phi_{N}^{\prime}\left(\bar{x}_{k}\right) v_{N} \\
& +\left(4 \sum_{k=1}^{n} \beta_{k}^{2} \phi_{N}^{4}\left(\bar{x}_{k}\right)-2 \sum_{k=1}^{n} \beta_{k} \gamma^{-1 / 2} A_{k} \phi_{N}^{2}\left(\bar{x}_{k}\right)\right) v_{N} \\
& +\psi_{N} \gamma^{-1} F .
\end{aligned}
$$

Multiplying the both sides of (30) by $v_{N}$ and integrating over $\mathbf{R}^{n}$, we obtain

$\partial_{\bar{t}}\left\|v_{N}\right\|_{L^{2}\left(\mathbf{R}^{n}\right)}^{2}$

$$
\begin{aligned}
& =\int \Delta v_{N} v_{N} d \bar{x}-\int \gamma^{-1 / 2} A \cdot \nabla v_{N} v_{N} d \bar{x}-\int \gamma^{-1} B\left(v_{N}\right) v_{N} d \bar{x} \\
& -4 \int\left(\sum_{k=1}^{n} \beta_{k} \phi_{N}^{2}\left(\bar{x}_{k}\right) \partial_{\bar{x}_{k}} v_{N}\right. \\
& \left.+\sum_{k=1}^{n} \beta_{k} \phi_{N}\left(\bar{x}_{k}\right) \phi_{N}^{\prime}\left(\bar{x}_{k}\right) v_{N}\right) v_{N} d \bar{x} \\
& +\int\left(4 \sum_{k=1}^{n} \beta_{k}^{2} \phi_{N}^{4}\left(\bar{x}_{k}\right)-2 \sum_{k=1}^{n} \beta_{k} \gamma^{-1 / 2} A_{k} \phi_{N}^{2}\left(\bar{x}_{k}\right)\right) v_{N} v_{N} d \bar{x} \\
& +\int \psi_{N} \gamma^{-1} F v_{N} d \bar{x} .
\end{aligned}
$$

Using the integration by parts, it follows that

$$
\begin{aligned}
\partial_{\bar{t}}\left\|v_{N}\right\|^{2} & \\
\leq & -\left\|\nabla v_{N}\right\|^{2}+\gamma^{-1 / 2}\|A\|_{L^{\infty}\left(\mathbf{R}^{n} \times[0,1]\right)}\left\|\nabla v_{N}\right\|\left\|v_{N}\right\| \\
& +\gamma^{-1}\|B\|_{L^{\infty}\left(\mathbf{R}^{n} \times[0,1]\right)}\left\|v_{N}\right\|^{2} \\
& +4|\beta|\left\|\nabla v_{N}\right\|\left\|v_{N}\right\| \quad(\text { by }(26)) \\
& +\left(4|\beta|^{2}+2 \sum_{k=1}^{n} \beta_{k} \gamma^{-1 / 2}\left\|A_{k}\right\|_{L^{\infty}\left(\mathbf{R}^{n} \times[0,1]\right)}\right)\left\|v_{N}\right\|^{2} \\
& +\gamma^{-1}\left\|\psi_{N} F\right\|\left\|v_{N}\right\| .
\end{aligned}
$$


Using the Young inequality, we have

$$
\begin{aligned}
& \partial_{\bar{t}}\left\|v_{N}\right\|^{2} \\
& \leq\left(13|\beta|^{2}+\gamma^{-1}\|B\|_{L^{\infty}\left(\mathbf{R}^{n} \times[0,1]\right)}\right. \\
& \left.\quad+\frac{\gamma^{-1}\left(3\|A\|_{L^{\infty}\left(\mathbf{R}^{n} \times[0,1]\right)}^{2}+1\right)}{2}\right)\left\|v_{N}\right\|^{2}+\frac{\gamma^{-1}\left\|\psi_{N} F\right\|^{2}}{2} .
\end{aligned}
$$

Denote by

$$
\begin{aligned}
a(\beta)= & 13|\beta|^{2}+\gamma^{-1}\|B\|_{L^{\infty}\left(\mathbf{R}^{n} \times[0,1]\right)} \\
& +\frac{\gamma^{-1}\left(3\|A\|_{L^{\infty}\left(\mathbf{R}^{n} \times[0,1]\right)}^{2}+1\right)}{2} .
\end{aligned}
$$

Integrating $(33)$ over $(0, \bar{t})$, we obtain

$$
\left\|v_{N}(\bar{t})\right\|^{2} \leq\left\|v_{N}(0)\right\|^{2}+e^{a(\beta)} \gamma^{-1} \int_{0}^{\bar{t}} \frac{\left\|\psi_{N} F\right\|^{2}}{2} d \bar{t} .
$$

Letting $N \rightarrow \infty$, we obtain that

$$
\int e^{2 \beta \cdot \bar{x}}|\bar{q}(\bar{t})|^{2} d \bar{x} \leq \int e^{2 \beta \cdot \bar{x}}|\bar{q}(0)|^{2} d \bar{x}+e^{a(\beta)} \int_{0}^{\bar{t}} \frac{\left\|e^{\beta \cdot \bar{x}} F\right\|^{2}}{2} d \bar{t} .
$$

The above variables $\bar{x}, \bar{t}$ are changed to $x, t$; we get

$$
\begin{aligned}
& \int e^{2 \beta \cdot \gamma^{1 / 2} x}|q(t)|^{2} d x \\
& \quad \leq \int e^{2 \beta \cdot \gamma^{1 / 2} x}|q(0)|^{2} d x+e^{a(\beta)} \gamma^{-1} \int_{0}^{1} \frac{\left\|e^{\beta \cdot \gamma^{1 / 2} x} F\right\|^{2}}{2} d t .
\end{aligned}
$$

On the other hand, it is easy to prove that

$$
e^{\gamma|x|^{2} / K}=\left(\frac{K}{\pi}\right)^{n / 2} \int e^{2 \beta \cdot \gamma^{1 / 2} x-K|\beta|^{2}} d \beta .
$$

In fact, noticing that

$$
\int_{\mathbf{R}^{n}} e^{-|x|^{2}} d x=\pi^{n / 2}
$$

and using

$$
2 \beta \cdot \gamma^{1 / 2} x-K|\beta|^{2}=-K\left|\beta-\frac{\gamma^{1 / 2} x}{K}\right|^{2}+\frac{\gamma|x|^{2}}{K},
$$

we have

$$
\begin{aligned}
& \left(\frac{K}{\pi}\right)^{n / 2} \int e^{2 \beta \cdot \gamma^{1 / 2} x-K|\beta|^{2}} d \beta \\
& \quad=\left(\frac{K}{\pi}\right)^{n / 2} \int e^{-K\left|\beta-\gamma^{1 / 2} x / K\right|^{2}+\gamma|x|^{2} / K} d \beta \\
& =e^{\gamma|x|^{2} / K} .
\end{aligned}
$$

That is, (38) holds.
Using (37) and (38), we have

$$
\begin{aligned}
& \int e^{\gamma|x|^{2} / K}|q(x, t)|^{2} d x \\
& =\left(\frac{K}{\pi}\right)^{n / 2} \iint e^{2 \beta \cdot \gamma^{1 / 2} x-K|\beta|^{2}} d \beta|q(x, t)|^{2} d x \\
& \leq\left(\frac{K}{\pi}\right)^{n / 2} \iint e^{-K|\beta|^{2}} e^{2 \beta \cdot \gamma^{1 / 2} x}|q(x, 0)|^{2} d \beta d x \\
& +\left(\frac{K}{\pi}\right)^{n / 2} \int_{0}^{1} \iint \frac{e^{a(\beta)-K|\beta|^{2}} e^{2 \beta \cdot \gamma^{1 / 2} x}|F|^{2}}{2} d \beta d x d t \\
& =\left(\frac{K}{\pi}\right)^{n / 2} \cdot \iint e^{-(K-13)\left|\beta-\gamma^{1 / 2} x /(K-13)\right|^{2}+\gamma|x|^{2} /(K-13)} \\
& \times|q(x, 0)|^{2} d \beta d x \\
& +\left(\frac{K}{\pi}\right)^{n / 2} e^{\gamma^{-1}\left(2\|B\|_{L^{\infty}\left(\mathbb{R}^{n} \times[0,1]\right)}+3\|A\|_{L^{\infty}\left(\mathbb{R}^{n} \times[0,1]\right)}^{2}+1\right) / 2} \\
& \times \int_{0}^{1} \iint \frac{e^{-(K-13)\left|\beta-\gamma^{1 / 2} x /(K-13)\right|^{2}+\gamma|x|^{2} /(K-13)}|F|^{2}}{2} d \beta d x d t \\
& =\left(\frac{K}{(K-13)}\right)^{n / 2} \cdot \int e^{\gamma|x|^{2} /(K-13)}|q(x, 0)|^{2} d x
\end{aligned}
$$

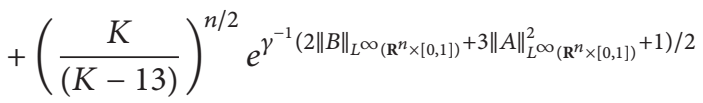

$$
\begin{aligned}
& \times \int_{0}^{1} \int^{\gamma e^{\gamma|x|^{2} /(K-13)}|F|^{2}}-2 x d t .
\end{aligned}
$$

In (42), taking $K=14$, it follows that

$$
\begin{aligned}
\int e^{\gamma|x|^{2} / 14}|q(x, t)|^{2} d x \\
\leq 14^{n / 2} \int e^{\gamma|x|^{2}}|q(x, 0)|^{2} d x \\
+14^{n / 2} e^{\left.\gamma^{\gamma^{-1}\left(2\|B\|_{L^{\infty}}\left(\mathbf{R}^{n} \times[0,1]\right)\right.}+3\|A\|_{L^{\infty}\left(\mathbf{R}^{n} \times[0,1]\right.}^{2}+1\right) / 2} \\
\quad \times \int_{0}^{1} \int \frac{e^{\gamma|x|^{2}}|F|^{2}}{2} d x d t .
\end{aligned}
$$

Replacing $\gamma / 14$ by $\gamma$ in (43) yields

$$
\begin{aligned}
\int e^{\gamma|x|^{2}} & |q(x, t)|^{2} d x \\
\leq & 14^{n / 2} \int e^{14 \gamma|x|^{2}}|q(x, 0)|^{2} d x \\
& +14^{n / 2} e^{(14 \gamma)^{-1}\left(2\|B\|_{L^{\infty}\left(\mathbb{R}^{n} \times[0,1]\right)}+3\|A\|_{L_{\left(R^{n} \times[0,1]\right)}^{2}}^{2}+1\right) / 2} \\
& \times \int_{0}^{1} \int \frac{e^{14 \gamma|x|^{2}}|F|^{2}}{2} d x d t .
\end{aligned}
$$

That is, (23) holds. The proof of Proposition 2 is completed. 
Remark 3. Taking the gradient operator $\nabla$ to the both sides of (21), we get the systems about $\nabla q$,

$$
\begin{aligned}
(\nabla q)_{t} & -\Delta(\nabla q) \\
& +(A \cdot \nabla)(\nabla q)+B(\nabla q)-((\nabla A) \cdot \nabla) q=(\nabla B) q,
\end{aligned}
$$

where replace $A, B$ by $A\left(\gamma^{-1 / 2} \bar{x}, \gamma^{-1} \bar{t}\right), B\left(\gamma^{-1 / 2} \bar{x}, \gamma^{-1} \bar{t}\right)$. Using Proposition 2, we get the estimates of the $\nabla q$,

$$
\begin{aligned}
\int e^{\gamma|x|^{2}}|\nabla q(x, t)|^{2} d x \\
\leq 14^{n / 2} \cdot \int e^{14 \gamma|x|^{2}}|\nabla q(x, 0)|^{2} d x \\
\quad+14^{n} e^{a\left(C_{0}, \gamma\right)}(14 \gamma)^{-1} \cdot\|\nabla B\|_{L^{\infty}} \int e^{196 \gamma|x|^{2}} \frac{|q(x, 0)|^{2}}{2} d x,
\end{aligned}
$$

where $\gamma>0, a\left(C_{0}, \gamma\right)=(14 \gamma)^{-1}\left(3 C_{0}^{2}+4 C_{0}+1\right) / 2$.

Proposition 2 shows that $\left\|e^{\gamma|x|^{2}} q\right\|$ relates to the initial value of $\left\|e^{14 \gamma|x|^{2}} q\right\|$, and Remark 3 means that $\left\|e^{\gamma|x|^{2}} \nabla q\right\|$ relates to the initial value of $\left\|e^{\gamma|x|^{2}} \nabla q\right\|$ and $\left\|e^{196 \gamma|x|^{2}} q\right\|$. But the key of this paper is to control the behavior of solution in interval $[0,1]$ by solution at two different times $t=0,1$. The following Lemma introduces an abstract result (for the tensor $v$ ) which shows how to get the "logarithmic convexity" property, which is analogous to Lemma 2 in [31] (for the complex function).

Lemma 4. Let $\mathcal{S}$ be a symmetric operator and $\mathscr{A}$ a skewsymmetric operator, both allowed to depend on the time variable. $G$ is a positive function; $v(x, t)$ is a reasonable real 2-order tensor function.

Denote

$$
\begin{gathered}
F(t)=(v(\cdot, t), v(\cdot, t)), \quad D(t)=(\mathcal{S} v, v), \\
E(t)=\frac{D(t)}{F(t)} .
\end{gathered}
$$

Moreover, if there exist constants $M_{0}, M_{1}$, and $M_{2}$ such that

$$
\begin{gathered}
\left|\partial_{t} v-\mathscr{A} v-\mathcal{S} v\right| \leq M_{1}\|v\|+G(t), \quad \mathcal{S}_{t}+[\mathcal{S}, \mathscr{A}] \geq-M_{0}, \\
M_{2}=\sup _{[0,1]} \frac{\|G(t)\|}{\|v(t)\|}
\end{gathered}
$$

are achieved, then $\log F(t)$ is "logarithmically convex" in $[0,1]$ and there is a universal constant $K$ such that

$$
F(t) \leq e^{K\left(M_{0}+M_{1}+M_{2}+M_{1}^{2}+M_{2}^{2}\right)} F(0)^{1-t} F(1)^{t} \quad \text { for } 0 \leq t \leq 1 .
$$

Proof. On one hand,

$$
\begin{gathered}
F^{\prime}(t)=2\left(\partial_{t} v, v\right)=\left(\partial_{t} v+\delta \mathcal{S} v\right)+\left(\partial_{t} v-\delta v, v\right), \\
D(t)=\frac{1}{2}\left(\partial_{t} v+\delta v, v\right)-\frac{1}{2}\left(\partial_{t} v-\delta v, v\right)
\end{gathered}
$$

Because $\mathscr{A}$ is a skew-symmetric operator and $(\mathscr{A} v, v)=0$, thus

$$
\begin{aligned}
F^{\prime}(t) D(t) & =\frac{1}{2}\left(\partial_{t} v+\mathcal{S} v, v\right)^{2}-\frac{1}{2}\left(\partial_{t} v-\mathcal{S} v, v\right)^{2} \\
& =\frac{1}{2}\left(\partial_{t} v-\mathscr{A} v+\mathcal{S} v, v\right)^{2}-\frac{1}{2}\left(\partial_{t} v-\mathscr{A} v-\mathcal{S} v, v\right)^{2}
\end{aligned}
$$

On the other hand

$$
\begin{aligned}
F^{\prime}(t) & =2\left(\partial_{t} v, v\right)=2\left(\partial_{t} v-\delta v-\mathscr{A} v, v\right)+2(\delta, v) \\
& =2\left(\partial_{t} v-\delta v-\mathscr{A} v, v\right)+2 D(t)
\end{aligned}
$$

Differentiating $D(t)=(\mathcal{S} v, v)$, we get

$$
\begin{aligned}
D^{\prime}(t) & =\left(\mathcal{S}_{t} v, v\right)+\left(\mathcal{S} \partial_{t} v, v\right)+\left(\mathcal{S} v, \partial_{t} v\right) \\
& =\left(\mathcal{S}_{t} v+[\mathcal{S}, \mathscr{A}] v, v\right)+2\left(\partial_{t} v-\mathscr{A} v, \mathcal{S} v\right) .
\end{aligned}
$$

Noticing that

$$
\begin{aligned}
2\left(\partial_{t} v\right. & -\mathscr{A} v, \mathcal{S} v) \\
& =\frac{1}{2}\left\|\partial_{t} v-\mathscr{A} v+\mathcal{S} v\right\|^{2}-\frac{1}{2}\left\|\partial_{t} v-\mathscr{A} v-\mathcal{S} v\right\|^{2},
\end{aligned}
$$

we have

$$
\begin{aligned}
D^{\prime}(t)= & \left(\mathcal{S}_{t} v+[\mathcal{S}, \mathscr{A}] v, v\right) \\
& +\frac{1}{2}\left\|\partial_{t} v-\mathscr{A} v+\mathcal{S} v\right\|^{2}-\frac{1}{2}\left\|\partial_{t} v-\mathscr{A} v-\mathcal{S} v\right\|^{2} .
\end{aligned}
$$

From (52) and (55), it follows that

$$
\begin{gathered}
F^{\prime \prime}(t)=2 \partial_{t}\left(\partial_{t} v-\mathcal{S} v-\mathscr{A} v, v\right)+2\left(\mathcal{S}_{t} v+[\mathcal{S}, \mathscr{A}] v, v\right) \\
+\left\|\partial_{t} v-\mathscr{A} v+\mathcal{S} v\right\|^{2}-\left\|\partial_{t} v-\mathcal{S} v-\mathscr{A} v\right\|^{2} .
\end{gathered}
$$

From (55) and (51), it follows that

$E^{\prime}(t)$

$$
\begin{aligned}
= & \frac{\left(\mathcal{S}_{t} v+[\mathcal{S}, \mathscr{A}] v, v\right)}{F} \\
& +\frac{(1 / 2)\left[\left\|\partial_{t} v-\mathscr{A} v+\mathcal{S} v\right\|^{2}\|v\|^{2}-\left(\partial_{t} v-\mathscr{A} v+\mathcal{S} v, v\right)^{2}\right]}{F^{2}} \\
& +\frac{(1 / 2)\left[\left(\partial_{t} v-\mathscr{A} v-\mathcal{S} v, v\right)^{2}-\left\|\partial_{t} v-\mathscr{A} v-\mathcal{S} v\right\|^{2}\|v\|^{2}\right]}{F^{2}} .
\end{aligned}
$$

Using the Cauchy-Schwarz inequality, we have

$$
\left\|\partial_{t} v-\mathscr{A} v+\delta v\right\|^{2}\|v\|^{2}-\left(\partial_{t} v-\mathscr{A} v+\delta v, v\right)^{2} \geq 0 .
$$

Meantime, $(1 / 2)\left(\partial_{t} v-\mathscr{A} v-\delta v, v\right)^{2}>0$, so

$$
E^{\prime}(t) \geq \frac{\left(\mathcal{S}_{t} v+[\mathcal{S}, \mathscr{A}] v, v\right)}{F}-\frac{\left\|\partial_{t} v-\mathscr{A} v-\mathcal{S} v\right\|^{2}}{(2 F)}
$$


From (48) and the inequality above,

$$
E^{\prime}(t) \geq-M_{0}-M_{1}^{2}-M_{2}^{2}
$$

We remark (52) shows that

$$
\partial_{t}[\log F(t)+O(1)]=2 E(t),
$$

where $O(1)$ is a function and

$$
\begin{array}{r}
|O(1)| \leq K\left(M_{0}+M_{1}+M_{2}+M_{1}^{2}+M_{2}^{2}\right), \\
\text { when } 0 \leq t \leq 1 .
\end{array}
$$

All together, when $0 \leq t \leq 1$,

$$
\partial_{t}^{2}(\log F(t)+O(1)) \geq 0, \quad \text { when } 0 \leq t \leq 1 .
$$

Therefore, when $0 \leq s \leq t \leq \lambda \leq 1$,

$$
\partial_{s}(\log F(s)+O(1)) \leq \partial_{\lambda}(\log F(\lambda)+O(1)) .
$$

On one hand, the integration of the inequality above over the intervals $0 \leq s \leq t$ and $t \leq \lambda \leq 1$ shows that

$$
\begin{aligned}
& \int_{t}^{1} \int_{0}^{t} \partial_{s}(\log F(s)+O(1)) d s d \lambda \\
& \quad=\int_{t}^{1}(\log F(t)-\log F(0)+O(1) t) d \lambda \\
& \quad=(1-t) \log F(t)-(1-t) \log F(0)+O(1) t(1-t) .
\end{aligned}
$$

On the other hand,

$$
\begin{aligned}
& \int_{0}^{t} \int_{t}^{1} \partial_{\lambda}(\log F(\lambda)+O(1)) d \lambda d s \\
& \quad=\int_{0}^{t}(\log F(1)-\log F(t)+O(1)(1-t) d s) \\
& \quad=t \log F(1)-t \log F(t)+O(1) t(1-t) .
\end{aligned}
$$

Combining with (64), it implies (49). This completes the proof of Lemma 4.

Proposition 5. Assume that $q \in L^{\infty}\left([0,1], L^{2}\left(\mathbf{R}^{n}\right)\right) \bigcap L^{2}$ $\left([0,1], H^{1}\left(\mathbf{R}^{n}\right)\right)$ verifies (21) and $\left\|e^{196 \gamma|x|^{2}} q(0)\right\|_{L^{2}\left(\mathbf{R}^{n}\right)}$, $\left\|e^{196 \gamma|x|^{2}} q(1)\right\|_{L^{2}\left(\mathbf{R}^{n}\right)}$ are finite as $\gamma>5 C_{0} / 16$, and let

$$
M_{0}=8 \gamma^{2} C_{1}+2 C_{0},
$$$$
\|B\|_{L^{\infty}\left(\mathbf{R}^{n} \times[0,1]\right)}+2 \gamma\|A \cdot x\|_{L^{\infty}\left(\mathbf{R}^{n} \times[0,1]\right)} \leq C_{0}+2 \gamma C_{1} \leq M_{1} .
$$

Then, $\left\|e^{\gamma|x|^{2}} q(t)\right\|_{L^{2}\left(\mathbf{R}^{n}\right)}$ is "logarithmical convexity" in $[0,1]$ and there is a universal constant $K$ such that

$$
\left\|e^{\gamma|x|^{2}} q(t)\right\| \leq e^{K\left(M_{0}+M_{1}+M_{1}{ }^{2}\right)}\left\|e^{\gamma|x|^{2}} q(0)\right\|^{1-t}\left\|e^{\gamma|x|^{2}} q(1)\right\|^{t},
$$

when $0 \leq t \leq 1$
Proof. Let $v=e^{\gamma \varphi} q$; then, from the equation about $q$, we get the equation

$$
\begin{aligned}
\partial_{t} v= & \left(\Delta+\gamma^{2}|\nabla \varphi|^{2}+\gamma \partial_{t} \varphi\right) v \\
& +(-2 \gamma \nabla \varphi \cdot \nabla-\gamma \Delta \varphi-A \cdot \nabla) v+(\gamma A \cdot \nabla \varphi-B) v \\
= & \delta v+\mathscr{A} v+(\gamma A \cdot \nabla \varphi-B) v,
\end{aligned}
$$

where $\mathcal{S}=\Delta+\gamma^{2}|\nabla \varphi|^{2}+\gamma \partial_{t} \varphi$ is a symmetric operator and $\mathscr{A}=-2 \gamma \nabla \varphi \cdot \nabla-\gamma \Delta \varphi-A \cdot \nabla$ is a skew-symmetric operator. At this time,

$$
\mathcal{S}_{t}=2 \gamma^{2} \nabla \varphi \cdot \nabla \partial_{t} \varphi+\gamma \partial_{t}^{2} \varphi
$$

Let $A^{i}$ be the $i$ th element of $A$ and $\partial_{i} v$ the partial derivative on $x_{i}$. A calculation shows that

$$
\begin{aligned}
\left(\mathcal{S}_{t}+\right. & {[\mathcal{S}, \mathscr{A}]) v } \\
= & \left(\gamma \partial_{t}^{2} \varphi+4 \gamma^{2} \nabla \varphi \cdot \nabla \partial_{t} \varphi\right) v \\
& -\gamma\left(4 \nabla \cdot\left(D^{2} \varphi \nabla\right)-4 \gamma^{2} D^{2} \varphi \nabla \varphi \cdot \nabla \varphi+\Delta^{2} \varphi\right) v \\
& +\gamma\left(A \cdot \nabla \partial_{t} \varphi\right) v+\gamma^{2}\left(A \cdot \nabla\left(|\nabla \varphi|^{2}\right)\right) v \\
& -\sum_{i=1}^{n}\left(\Delta A^{i}\right) \partial_{i} v-2 \sum_{i=1}^{n}\left(\nabla A^{i} \cdot \nabla\right) \partial_{i} v .
\end{aligned}
$$

Thus, if we take $\varphi=|x|^{2}$,

$\left(\left(\mathcal{S}_{t}+[\mathcal{S}, \mathscr{A}]\right) v, v\right)$

$$
\begin{gathered}
=8 \gamma \int_{\mathbf{R}^{n}}|\nabla v|^{2} d x+32 \gamma^{3} \int_{\mathbf{R}^{n}}|x|^{2}|v|^{2} d x+8 \gamma^{2} \int_{\mathbf{R}^{n}} A \cdot x|v|^{2} d x \\
-\int_{\mathbf{R}^{n}} \sum_{i=1}^{n}\left(\Delta A^{i}\right) \partial_{i} v v d x-2 \int_{\mathbf{R}^{n}} \sum_{i=1}^{n}\left(\nabla A^{i} \cdot \nabla\right) \partial_{i} v v d x .
\end{gathered}
$$

A formal integration by parts shows that

$$
\begin{aligned}
& -2 \int_{\mathbf{R}^{n}} \sum_{i=1}^{n}\left(\nabla A^{i} \cdot \nabla\right) \partial_{i} v v d x \\
& \quad=2 \int_{\mathbf{R}^{n}}(\Delta A \cdot \nabla v) v d x+2 \int_{\mathbf{R}^{n}}(\nabla A \cdot \nabla v) \cdot \nabla v d x, \\
& -\int_{\mathbf{R}^{n}} \sum_{i=1}^{n}\left(\Delta A^{i}\right) \partial_{i} v v d x=-\int_{\mathbf{R}^{n}}(\Delta A \cdot \nabla v) v d x .
\end{aligned}
$$


Using the two results above and noticing that $\|A \cdot x\|_{\infty} \leq C_{1}$ and $\|\nabla A\|_{\infty},\|\nabla A\|_{\infty} \leq C_{0}$, we get that

$$
\begin{aligned}
& \left(\left(\mathcal{S}_{t}+[\mathcal{S}, \mathscr{A}]\right) v, v\right) \\
& =8 \gamma \int_{\mathbf{R}^{n}}|\nabla v|^{2} d x+32 \gamma^{3} \int_{\mathbf{R}^{n}}|x|^{2}|v|^{2} d x+8 \gamma^{2} \int_{\mathbf{R}^{n}} A \cdot x|v|^{2} d x \\
& \quad+\int_{\mathbf{R}^{n}}(\Delta A \cdot \nabla v) v d x+2 \int_{\mathrm{R}^{n}}(\nabla A \cdot \nabla v) \cdot \nabla v d x \\
& \geq 8 \gamma \int_{\mathbf{R}^{n}}|\nabla v|^{2} d x+32 \gamma^{3} \int_{\mathbf{R}^{n}}|x|^{2}|v|^{2} d x-8 \gamma^{2} C_{1} \int_{\mathbf{R}^{n}}|v|^{2} d x \\
& \quad-\frac{C_{0}}{2} \int_{\mathbf{R}^{n}}|\nabla v|^{2} d x-2 C_{0} \int_{\mathbf{R}^{n}}|v|^{2} d x-2 C_{0} \int_{\mathbf{R}^{n}}|\nabla v|^{2} d x \\
& =\left(8 \gamma-\frac{C_{0}}{2}-2 C_{0}\right) \int_{\mathrm{R}^{n}}|\nabla v|^{2} d x+32 \gamma^{3} \\
& \quad \times \int_{\mathbf{R}^{n}}|x|^{2}|v|^{2} d x-\left(8 \gamma^{2} C_{1}+2 C_{0}\right) \int_{\mathbf{R}^{n}}|v|^{2} d x .
\end{aligned}
$$

When $\gamma>5 C_{0} / 16$, we have

$$
\begin{aligned}
\left(\left(\mathcal{S}_{t}+[\mathcal{S}, \mathscr{A}]\right) v, v\right) & \geq-\left(8 \gamma^{2} C_{1}+2 C_{0}\right) \int_{\mathbf{R}^{n}}|v|^{2} d x \\
& =-M_{0} \int_{\mathbf{R}^{n}}|v|^{2} d x .
\end{aligned}
$$

So, $\mathcal{S}_{t}+[\mathcal{S}, \mathscr{A}] \geq-M_{0}$. On the other hand, we get

$$
\begin{aligned}
\left|\partial_{t} v-\mathcal{S} v-\mathscr{A} v\right| & =|(\gamma A \cdot \nabla \varphi-B) v| \\
& \leq\left(\|B\|_{L^{\infty}}+2 \gamma\|A \cdot x\|_{L^{\infty}}\right)\|v\| \\
& \leq\left(C_{0}+2 \gamma C_{1}\right)\|v\| \leq M_{1}\|v\| .
\end{aligned}
$$

Therefore when $v=e^{\gamma|x|^{2}} q$, from Lemma 4, we have the "logarithmic convexity" of $F(t)=\left\|e^{\gamma|x|^{2}} q(t)\right\|^{2}$ and then (68) follows. Proposition 2 shows the validity of the previous arguments. This completes the proof of Proposition 5.

Proposition 6. Assume that $q, A, B$, and $\gamma$ are as in Proposition 5 and $\gamma>0$ is finite; then,

$$
\begin{aligned}
& \left\|\sqrt{t(1-t)} e^{\gamma|x|^{2}} \nabla q\right\|_{L^{2}\left(\mathbf{R}^{n} \times[0,1]\right)} \\
& \quad+\left\|\sqrt{t(1-t)}|x| e^{\gamma|x|^{2}} q\right\|_{L^{2}\left(\mathbf{R}^{n} \times[0,1]\right)} \\
& \leq K\left(M_{0}+M_{1}+M_{1}^{2}\right) \sup _{[0,1]}\left\|e^{\gamma|x|^{2}} q(t)\right\|,
\end{aligned}
$$

where $K, M_{0}$, and $M_{1}$ are as in Proposition 5.
Proof. Let $v=e^{\gamma|x|^{2}} q$, from Proposition 5; we have

$$
\begin{gathered}
\left|\partial_{t} v-\delta \mathcal{S} v \mathscr{A} v\right| \leq M_{1}\|v\|, \\
\left(\left(\mathcal{S}_{t}+[\mathcal{S}, \mathscr{A}]\right) v, v\right) \\
\geq\left(8 \gamma-\frac{C_{0}}{2}-2 C_{0}\right) \int_{\mathbf{R}^{n}}|\nabla v|^{2} d x \\
\quad+32 \gamma^{3} \int_{\mathbf{R}^{n}}|x|^{2}|v|^{2} d x-\left(8 \gamma^{2} C_{1}+2 C_{0}\right) \int_{\mathbf{R}^{n}}|v|^{2} d x \\
=P_{1} \int_{\mathbf{R}^{n}}|\nabla v|^{2} d x+P_{2} \int_{\mathbf{R}^{n}}|x|^{2}|v|^{2} d x-P_{3} \int_{\mathbf{R}^{n}}|v|^{2} d x,
\end{gathered}
$$

where $P_{1}=8 \gamma-\left(5 C_{0} / 2\right), P_{2}=32 \gamma^{3}$, and $P_{3}=8 \gamma^{2} C_{1}+2 C_{0}=$ $M_{0}$.

Thus, integration over $[0,1]$ to $t(1-t)$ timing $F^{\prime \prime}(t)$ of the formula (56) in Lemma 4 shows that

$$
\int_{0}^{1} t(1-t) F^{\prime \prime}(t) d t=F(1)+F(0)-2 \int_{0}^{1} F(t) d t .
$$

On the other hand, integrating the above equality by parts shows

$$
\begin{aligned}
\int_{0}^{1} 2 t & (1-t) \partial_{t}\left(\partial_{t} v-\mathcal{S} v-\mathscr{A} v, v\right) d t \\
& +\int_{0}^{1} 2 t(1-t)\left(\mathcal{S}_{t} v+[\mathcal{S}, \mathscr{A}] v, v\right) d t \\
& +\int_{0}^{1} t(1-t)\left\|\partial_{t} v-\mathscr{A} v+\mathcal{S} v\right\|^{2} d t \\
& -\int_{0}^{1} t(1-t)\left\|\partial_{t} v-\mathscr{A} v-\mathcal{S} v\right\|^{2} d t \\
\geq & -\int_{0}^{1}(2-4 t)\left(\partial_{t} v-\mathcal{S} v-\mathscr{A} v, v\right) d t \\
& +\int_{0}^{1} 2 t(1-t)\left(\mathcal{S}_{t} v+[\mathcal{S}, \mathscr{A}] v, v\right) d t \\
& -\int_{0}^{1} t(1-t)\left\|\partial_{t} v-\mathscr{A} v-\mathcal{S} v\right\|^{2} d t .
\end{aligned}
$$

Therefore

$$
\begin{aligned}
F(1) & +F(0)-2 \int_{0}^{1} F(t) d t \\
\geq & -\int_{0}^{1}(2-4 t)\left(\partial_{t} v-\mathcal{S} v-\mathscr{A} v, v\right) d t \\
& +\int_{0}^{1} 2 t(1-t)\left(\mathcal{S}_{t} v+[\mathcal{S}, \mathscr{A}] v, v\right) d t \\
& -\int_{0}^{1} t(1-t)\left\|\partial_{t} v-\mathscr{A} v-\mathcal{S} v\right\|^{2} d t
\end{aligned}
$$


that is,

$$
\begin{aligned}
& \int_{0}^{1} 2 t(1-t)\left(\mathcal{S}_{t} v+[\mathcal{S}, \mathscr{A}] v, v\right) d t+2 \int_{0}^{1} F(t) d t \\
& \leq F(1)+F(0)+\int_{0}^{1}(2-4 t)\left(\partial_{t} v-\mathcal{S} v-\mathscr{A} v, v\right) d t \\
& \quad+\int_{0}^{1} t(1-t)\left\|\partial_{t} v-\mathscr{A} v-\mathcal{S} v\right\|^{2} d t
\end{aligned}
$$

From (78) and (46), we obtain

$$
\begin{aligned}
& 2 \int_{0}^{1} t(1-t) \int_{\mathbf{R}^{n}} P_{1}|\nabla v|^{2}+P_{2}|x|^{2}|v|^{2}-P_{3}|v|^{2} d x d t \\
& \quad+2 \int_{0}^{1} F(t) d t \\
& \leq F(1)+F(0)+2 \int_{0}^{1} \int_{\mathbf{R}^{n}} M_{1}|v|^{2} d x d t \\
& \quad+\int_{0}^{1} \int_{\mathbf{R}^{n}} t(1-t) M_{1}^{2}|v|^{2} d x d t .
\end{aligned}
$$

To be simplified,

$$
\begin{aligned}
& 2 \int_{0}^{1} t(1-t) \int_{\mathbf{R}^{n}} P_{1}|\nabla v|^{2}+4 \gamma^{2} P_{1}|x|^{2}|v|^{2} d x d t \\
& \quad+2\left(P_{2}-4 \gamma^{2} P_{1}\right) \int_{0}^{1} t(1-t) \int_{\mathbf{R}^{n}}|x|^{2}|v|^{2} d x d t \\
& \quad \leq F(1)+F(0)+\left(2 M_{1}+\frac{M_{1}^{2}}{4}+\frac{P_{3}}{2}-2\right) \int_{0}^{1} \int_{\mathbf{R}^{n}}|v|^{2} d x d t \\
& \quad \leq F(1)+F(0)+\left(2 M_{1}+\frac{M_{1}^{2}}{4}+\frac{M_{0}}{2}\right) \int_{0}^{1} \int_{\mathbf{R}^{n}}|v|^{2} d x d t .
\end{aligned}
$$

Moreover, from $v=e^{\gamma|x|^{2}} q$, by Cauchy-Schwarz inequality and integration by parts, it is easy to obtain

$$
\begin{aligned}
& \int_{\mathbf{R}^{n}}|\nabla v|^{2}+4 \gamma^{2}|x|^{2}|v|^{2} d x \\
& \quad \geq 2 \gamma n \int_{\mathbf{R}^{n}}|v|^{2} d x=2 \gamma n \int_{\mathbf{R}^{n}} e^{2 \gamma|x|^{2}}|q|^{2} d x .
\end{aligned}
$$

Furtherly,

$$
\begin{aligned}
\int_{\mathbf{R}^{n}}|\nabla v|^{2}+4 \gamma^{2}|x|^{2}|v|^{2} d x \\
=\int_{\mathbf{R}^{n}} 8 \gamma^{2}|x|^{2} e^{2 \gamma|x|^{2}}|q|^{2}+e^{2 \gamma|x|^{2}}|\nabla q|^{2} d x \\
\quad+\int_{\mathbf{R}^{n}} 4 \gamma x e^{2 \gamma|x|^{2}} q \nabla q d x \\
=\int_{\mathbf{R}^{n}} 8 \gamma^{2}|x|^{2} e^{2 \gamma|x|^{2}}|q|^{2}+e^{2 \gamma|x|^{2}}|\nabla q|^{2} d x \\
\quad-2 \gamma n \int_{\mathbf{R}^{n}} e^{2 \gamma|x|^{2}}|q|^{2} d x-\int_{\mathbf{R}^{n}} 8 \gamma^{2}|x|^{2} e^{2 \gamma|x|^{2}}|q|^{2} d x \\
=\int_{\mathbf{R}^{n}} e^{2 \gamma|x|^{2}}|\nabla q|^{2} d x-2 \gamma n \int_{\mathbf{R}^{n}} e^{2 \gamma|x|^{2}}|q|^{2} d x .
\end{aligned}
$$

Thus, the last two formulae give

$$
2 \int_{\mathbf{R}^{n}}|\nabla v|^{2}+4 \gamma^{2}|x|^{2}|v|^{2} d x \geq \int_{\mathbf{R}^{n}} e^{2 \gamma|x|^{2}}|\nabla q|^{2} d x .
$$

Applying (87) to (84), we have

$$
\begin{aligned}
& P_{1} \int_{0}^{1} t(1-t) \int_{\mathbf{R}^{n}} e^{2 \gamma|x|^{2}}|\nabla q|^{2} d x \\
& \quad+2\left(P_{2}-4 \gamma^{2} P_{1}\right) \int_{0}^{1} t(1-t) \int_{\mathbf{R}^{n}}|x|^{2}|v|^{2} d x d t \\
& \quad \leq F(1)+F(0)+\left(2 M_{1}+\frac{M_{1}^{2}}{4}+\frac{M_{0}}{2}\right) \int_{0}^{1} \int_{\mathbf{R}^{n}}|v|^{2} d x d t
\end{aligned}
$$

Therefore, there is a constant $K$ such that

$$
\begin{aligned}
& \left\|\sqrt{t(1-t)} e^{\gamma|x|^{2}} \nabla q\right\|_{L^{2}\left(\mathbf{R}^{n} \times[0,1]\right)} \\
& \quad+\left\|\sqrt{t(1-t)}|x| e^{\gamma|x|^{2}} q\right\|_{L^{2}\left(\mathbf{R}^{n} \times[0,1]\right)} \\
& \leq K\left(M_{0}+M_{1}+M_{1}^{2}\right) \sup _{[0,1]}\left\|e^{\gamma|x|^{2}} q(t)\right\| .
\end{aligned}
$$

This completes the proof of Proposition 6.

\section{Carleman Estimates}

In this section, let $\gamma>5 C_{0} / 16$ and the assumptions in Propositions 5 and 6 satisfied; we get the following Carleman estimate.

Proposition 7 (Carleman Estimation). If $f \in C_{0}^{\infty}\left(\mathbf{R}^{n} \times\right.$ $[0,1])$,

$$
\begin{aligned}
\varphi= & \mu\left|x+R t(1-t) \vec{e}_{1}\right|^{2} \\
& +\frac{R^{2} t(1-t)(1-2 t)}{6}-\frac{(1+\varepsilon) R^{2} t(1-t)}{16 \mu},
\end{aligned}
$$


where $\vec{e}_{1}=(1,0, \ldots, 0)$, then, there exist $\varepsilon>0, \mu>0$, and $R>0$ such that

$R \sqrt{\frac{\varepsilon}{32 \mu}}\left\|e^{\varphi} f\right\|_{L^{2}\left(\mathbf{R}^{n} \times[0,1]\right)} \leq\left\|e^{\varphi}\left(\partial_{t}-\Delta+A \cdot \nabla\right) f\right\|_{L^{2}\left(\mathbf{R}^{n} \times[0,1]\right)}$.

Proof. Let $v=e^{\varphi} f$; then,

$$
\begin{aligned}
\left(\partial_{t}-\right. & \Delta+A \cdot \nabla) f \\
= & -\varphi_{t} e^{-\varphi} v+e^{-\varphi} \partial_{t} v+\Delta \varphi e^{-\varphi} v-|\nabla \varphi|^{2} e^{-\varphi} v \\
& +2 e^{-\varphi} \nabla \varphi \cdot \nabla v-e^{-\varphi} \Delta v-e^{-\varphi} v A \cdot \nabla \varphi+e^{-\varphi} A \cdot \nabla v .
\end{aligned}
$$

Therefore

$$
\begin{aligned}
e^{\varphi}\left(\partial_{t}-\Delta+A \cdot \nabla\right) f \\
=\partial_{t} v-\left(\Delta+|\nabla \varphi|^{2}+\partial_{t} \varphi+A \cdot \nabla \varphi\right) v \\
\quad+(2 \nabla \varphi \cdot \nabla+\Delta \varphi+A \cdot \nabla) \\
=\partial_{t} v-\delta \mathcal{S} v \mathscr{A} v
\end{aligned}
$$

where

$$
\begin{gathered}
\mathcal{S}=\Delta+|\nabla \varphi|^{2}+\varphi_{t}+A \cdot \nabla \varphi, \\
\mathscr{A}=-2 \nabla \varphi \cdot \nabla-\Delta \varphi-A \cdot \nabla \\
\mathcal{S}_{t}=2 \nabla \varphi \cdot \nabla \varphi_{t}+\varphi_{t t}+A_{t} \cdot \nabla \varphi+A \cdot \nabla \varphi_{t} .
\end{gathered}
$$

Set

$$
\begin{aligned}
& \mathcal{S}^{\prime}=\Delta+|\nabla \varphi|^{2}+\varphi_{t}, \\
& \mathscr{A}^{\prime}=-2 \nabla \varphi \cdot \nabla-\Delta \varphi, \\
& \mathcal{S}_{t}^{\prime}=2 \nabla \varphi \cdot \nabla \varphi_{t}+\varphi_{t t}
\end{aligned}
$$

then

$$
\begin{aligned}
{[\mathcal{S}, \mathscr{A}] } & v \\
= & \left(\mathcal{S}^{\prime}+A \cdot \nabla \varphi\right)\left(\mathscr{A}^{\prime} v-A \cdot \nabla v\right) \\
& +\left(-\mathscr{A}^{\prime}+A \cdot \nabla\right)\left(\mathcal{S}^{\prime} v+A \cdot \nabla \varphi v\right) \\
= & {\left[\mathcal{S}^{\prime}, \mathscr{A}^{\prime}\right] v-A \cdot \nabla \varphi A \cdot \nabla v+A \cdot \nabla(A \cdot \nabla \varphi v) } \\
& -2 A \cdot \nabla \varphi \nabla \varphi \cdot \nabla v+2 \nabla \varphi \cdot \nabla(A \cdot \nabla \varphi v) \\
& -\Delta(A \cdot \nabla v)+A \cdot \nabla(\Delta v) \\
& -|\nabla \varphi|^{2} A \cdot \nabla v+A \cdot \nabla\left(|\nabla \varphi|^{2} v\right)-\varphi_{t} A \cdot \nabla v+A \cdot \nabla\left(\varphi_{t} v\right) .
\end{aligned}
$$

So,

$$
\begin{aligned}
\left(\mathcal{S}_{t}+[\mathcal{S}, \mathscr{A}]\right) v= & \left(\mathcal{S}_{t}^{\prime}+\left[\mathcal{S}^{\prime}, \mathscr{A}^{\prime}\right]\right) v \\
& +A_{t} \cdot \nabla \varphi v+A \cdot \nabla \varphi_{t} v \\
& -A \cdot \nabla \varphi A \cdot \nabla v+A \cdot \nabla(A \cdot \nabla \varphi v) \\
& -2 A \cdot \nabla \varphi \nabla \varphi \cdot \nabla v+2 \nabla \varphi \cdot \nabla(A \cdot \nabla \varphi v) \\
& -\Delta(A \cdot \nabla v)+A \cdot \nabla(\Delta v) \\
& -|\nabla \varphi|^{2} A \cdot \nabla v+A \cdot \nabla\left(|\nabla \varphi|^{2} v\right) \\
& -\varphi_{t} A \cdot \nabla v+A \cdot \nabla\left(\varphi_{t} v\right)
\end{aligned}
$$

A calculation shows that

$$
\begin{aligned}
(99) & =[A \cdot \nabla(A \cdot \nabla \varphi)] v \\
(100) & =2 \nabla \varphi \cdot \nabla(A \cdot \nabla \varphi) v \\
(102) & =A \cdot \nabla\left(|\nabla \varphi|^{2}\right) v, \\
(103) & =\left(A \cdot \nabla \varphi_{t}\right) v, \\
(101) & =A \cdot \nabla(\Delta v)-\Delta(A \cdot \nabla v) \\
& =A \cdot \nabla(\Delta v)-\Delta\left(\sum_{i=1}^{n} A^{i} \partial_{i} v\right) \\
& =A \cdot \nabla(\Delta v)-\left[\sum_{i=1}^{n}\left(\Delta A^{i}\right) \partial_{i} v\right. \\
& =-\sum_{i=1}^{n}\left(\Delta A^{i}\right) \partial_{i} v-2 \sum_{i=1}^{n} \nabla A^{i} \cdot \nabla \partial_{i} v,
\end{aligned}
$$

where $A^{i}$ is the $i$ th element of $A, A_{t}^{i}$ is the partial derivatives on $t$ of $A^{i}$, and $\partial_{i} v$ is the partial derivatives on $v$ about $x_{i}$. That is,

$$
\begin{aligned}
\left(\mathcal{S}_{t}+\right. & {[\mathcal{S}, \mathscr{A}]) v } \\
= & \left(\mathcal{S}_{t}^{\prime}+\left[\mathcal{S}^{\prime}, \mathscr{A}^{\prime}\right]\right) v+\left(A_{t} \cdot \nabla \varphi\right) v \\
& +\left(A \cdot \nabla \varphi_{t}\right) v+(A \cdot \nabla(A \cdot \nabla \varphi)) v \\
& +2(\nabla \varphi \cdot \nabla(A \cdot \nabla \varphi)) v \\
& +\left(A \cdot \nabla\left(|\nabla \varphi|^{2}\right)\right) v+\left(A \cdot \nabla \varphi_{t}\right) v \\
& -\sum_{i=1}^{n}\left(\Delta A^{i}\right) \partial_{i} v-2 \sum_{i=1}^{n} \nabla A^{i} \cdot \nabla \partial_{i} v .
\end{aligned}
$$


So,

$$
\begin{aligned}
\left(\left(\mathcal{S}_{t}+\right.\right. & {[\mathcal{S}, \mathscr{A}]) v, v) } \\
= & \left(\left(\mathcal{S}_{t}^{\prime}+\left[\mathcal{S}^{\prime}, \mathscr{A}^{\prime}\right]\right) v, v\right)+\int_{\mathbf{R}^{n}}\left(A_{t} \cdot \nabla \varphi\right)|v|^{2} d x \\
& +2 \int_{\mathbf{R}^{n}}\left(A \cdot \nabla \varphi_{t}\right)|v|^{2} d x \\
& +\int_{\mathbf{R}^{n}}(A \cdot \nabla(A \cdot \nabla \varphi))|v|^{2} d x \\
& +2 \int_{\mathbf{R}^{n}}(\nabla \varphi \cdot \nabla(A \cdot \nabla \varphi))|v|^{2} d x \\
& +\int_{\mathbf{R}^{n}}\left(A \cdot \nabla\left(|\nabla \varphi|^{2}\right)\right)|v|^{2} d x-\int_{\mathbf{R}^{n}}\left(\sum_{i=1}^{n}\left(\Delta A^{i}\right) \partial_{i} v\right) v d x \\
& -2 \int_{\mathbf{R}^{n}}\left(\sum_{i=1}^{n} \nabla A^{i} \cdot \nabla \partial_{i} v\right) v d x .
\end{aligned}
$$

On one hand, a calculation implies

$$
\begin{aligned}
\mathcal{S}_{t}^{\prime}+\left[\mathcal{S}^{\prime}, \mathscr{A}^{\prime}\right]= & \varphi_{t t}+4 \nabla \varphi \cdot \nabla \varphi_{t}-4 \nabla \cdot\left(D^{2} \varphi \nabla\right) \\
& +4 D^{2} \varphi \nabla \varphi \cdot \nabla \varphi-\Delta^{2} \varphi
\end{aligned}
$$

and, from the definition of $\varphi$, we have

$$
\begin{aligned}
\mathcal{S}_{t}^{\prime} & +\left[\mathcal{S}^{\prime}, \mathscr{A}^{\prime}\right] \\
= & -4 \mu R\left(x_{1}+R t(1-t)\right)+2 \mu R^{2}(1-2 t)^{2}+(2 t-1) R^{2} \\
& \frac{(1+\varepsilon) R^{2}}{8 \mu}+4\left[\left(2 \mu x_{1}+2 \mu R t(1-t)\right)(2 \mu R(1-2 t))\right] \\
& -8 \mu \Delta+32 \mu^{3}\left|x+R t(1-t) \vec{e}_{1}\right|^{2} \\
= & -8 \mu \Delta+32 \mu^{3}\left|x+R t(1-t) \vec{e}_{1}\right|^{2} \\
& +2 \mu R^{2}(1-2 t)^{2}+(2 t-1) R^{2}+\frac{(1+\varepsilon) R^{2}}{8 \mu} \\
& +4 \mu R[4 \mu(1-2 t)-1]\left[x_{1}+R t(1-t)\right] \\
= & -8 \mu \Delta+\frac{\varepsilon R^{2}}{8 \mu}+32 \mu^{3}\left|x+R t(1-t) \vec{e}_{1}\right|^{2} \\
& +32 \mu^{3}\left[\frac{1}{32 \mu^{3}}\left(\frac{16 \mu^{2}(1-2 t)^{2} R^{2}-8 \mu(1-2 t) R^{2}+R^{2}}{8 \mu}\right)\right] \\
& +32 \mu^{3}\left[\frac{1}{32 \mu^{3}}\left(4 \mu R(4 \mu(1-2 t)-1)\left(x_{1}+R t(1-t)\right)\right)\right] \\
= & -8 \mu \Delta+\frac{\varepsilon R^{2}}{8 \mu}
\end{aligned}
$$

$$
\begin{gathered}
+32 \mu^{3}\left[\left|x+R t(1-t) \vec{e}_{1}\right|^{2}+\frac{(4 \mu(1-2 t)-1)^{2} R^{2}}{256 \mu^{4}}\right. \\
\left.+\frac{\left(x+R t(1-t) e_{1}\right)(4 \mu(1-2 t)-1) R \vec{e}_{1}}{8 \mu^{2}}\right] \\
=-8 \mu \Delta+\frac{\varepsilon R^{2}}{8 \mu} \\
+32 \mu^{3}\left|x+R t(1-t) \vec{e}_{1}+\frac{(4 \mu(1-2 t)-1) R \vec{e}_{1}}{16 \mu^{2}}\right|^{2} .
\end{gathered}
$$

So,

$$
\begin{aligned}
& \left(\left(\mathcal{S}_{t}^{\prime}+\left[\mathcal{S}^{\prime}, \mathscr{A}^{\prime}\right]\right) v, v\right) \\
& =32 \mu^{3} \int_{\mathbf{R}^{n}}\left|x+R t(1-t) \vec{e}_{1}+\frac{(4 \mu(1-2 t)-1) R \vec{e}_{1}}{16 \mu^{2}}\right|^{2}|v|^{2} d x \\
& \quad+\frac{\varepsilon R^{2}}{8 \mu} \int_{\mathbf{R}^{n}}|v|^{2} d x+8 \mu \int_{\mathbf{R}^{n}}|\nabla v|^{2} d x \\
& \geq \frac{\varepsilon R^{2}}{8 \mu} \int_{\mathbf{R}^{n}}|v|^{2} d x+8 \mu \int_{\mathbf{R}^{n}}|\nabla v|^{2} d x .
\end{aligned}
$$

On the other hand, some estimates below hold from the definition of $\varphi$ :

$$
\begin{gathered}
\int_{\mathbf{R}^{n}}\left(A_{t} \cdot \nabla \varphi\right)|v|^{2} d x \\
=\int_{\mathbf{R}^{n}} A_{t} \cdot\left[2 \mu\left(x+R t(1-t) \vec{e}_{1}\right)\right]|v|^{2} d x \\
=\int_{\mathbf{R}^{n}}\left[2 \mu\left(A_{t} \cdot x\right)+2 \mu R t(1-t) A_{t}^{1}\right]|v|^{2} d x \\
\geq-\left(2 \mu C_{1}+\frac{1}{2} \mu R C_{0}\right) \int_{\mathbf{R}^{n}}|v|^{2} d x, \\
\left.2 \int_{\mathbf{R}^{n}}\left(A \cdot \nabla \varphi_{t}\right)|v|^{2} d x \quad\left\|A_{t} \cdot x\right\|_{\infty} \leq C_{1},\left\|A_{t}^{1}\right\|_{\infty} \leq C_{0}\right), \\
=2 \int_{\mathbf{R}^{n}} 2 \mu R(1-2 t) A^{1}|v|^{2} d x \\
\geq-4 \mu R C_{0} \int_{\mathbf{R}^{n}}|v|^{2} d x, \quad\left(\text { by }\left\|A_{t}\right\|_{\infty} \leq C_{0}\right), \\
\int_{\mathbf{R}^{n}}(A \cdot \nabla(A \cdot \nabla \varphi))|v|^{2} d x \\
=\int_{\mathbf{R}^{n}}\left(A \cdot \nabla\left(A \cdot 2 \mu(x+R t(1-t)) \vec{e}_{1}\right)\right)|v|^{2} d x
\end{gathered}
$$




$$
\begin{aligned}
& =2 \mu \int_{\mathbf{R}^{n}}\left(A \cdot \nabla(A \cdot x)+R t(1-t) A \cdot \nabla A^{1}\right)|v|^{2} d x \\
& =2 \mu \int_{\mathbf{R}^{n}}\left(A \cdot(\nabla A \cdot x+A \cdot \nabla x)+R t(1-t) A \cdot \nabla A^{1}\right)|v|^{2} d x \\
& =2 \mu \int_{\mathbf{R}^{n}}\left(A \cdot x \cdot \nabla A+A \cdot A+R t(1-t) A \cdot \nabla A^{1}\right)|v|^{2} d x \\
& \geq-2 \mu\left(C_{1} C_{0}+C_{0}^{2}+\frac{R}{4} C_{0}^{2}\right) \int_{\mathbf{R}^{n}}|v|^{2} d x
\end{aligned}
$$

(by $\|A\|_{\infty} \leq C_{0},\|\nabla A\|_{\infty} \leq C_{0},\|A \cdot x\|_{\infty} \leq C_{1}$ ),

$2 \int_{\mathbf{R}^{n}}(\nabla \varphi \cdot \nabla(A \cdot \nabla \varphi))|v|^{2} d x$

$$
\begin{aligned}
& =2 \int_{\mathbf{R}^{n}} 2\left(\mu(x+R t(1-t)) \vec{e}_{1}\right) \\
& \cdot \nabla\left(A \cdot 2 \mu(x+R t(1-t)) \vec{e}_{1}\right)|v|^{2} d x \\
& =8 \mu^{2} \int_{\mathbf{R}^{n}}\left(x \cdot \nabla(A \cdot x)+R t(1-t) \partial_{1}(A \cdot x)\right. \\
& \left.\quad+R t(1-t) x \cdot \nabla A^{1}+R^{2} t^{2}(1-t)^{2} \partial_{1} A^{1}\right)|v|^{2} d x \\
& =8 \mu^{2} \int_{\mathbf{R}^{n}}\left((x \cdot \nabla A \cdot x)+x \cdot A+R t(1-t) \partial_{1} A \cdot x\right. \\
& \quad+R t(1-t) A^{1}+R t(1-t) x \\
& \left.\quad \cdot \nabla A^{1}+R^{2} t^{2}(1-t)^{2} \partial_{1} A^{1}\right)|v|^{2} d x \\
& \geq-8 \mu^{2}\left(C_{2}+C_{1}+\frac{R}{4}\left(2 C_{1}+C_{0}\right)+\frac{R^{2}}{4} C_{0}\right) \int_{\mathbf{R}^{n}}|v|^{2} d x,
\end{aligned}
$$

(by $\|x \cdot \nabla A \cdot x\|_{\infty} \leq C_{2},\|\nabla A\|_{\infty} \leq C_{0},\|A \cdot x\|_{\infty} \leq C_{1}$,

$$
\begin{aligned}
& \left.\|\nabla A \cdot x\|_{\infty} \leq C_{1},\|A\|_{\infty} \leq C_{0}\right), \\
& \int_{\mathbf{R}^{n}}\left(A \cdot \nabla\left(|\nabla \varphi|^{2}\right)\right)|v|^{2} d x \\
& =\int_{\mathbf{R}^{n}}\left(A \cdot \nabla\left(4 \mu^{2}\left|x+R t(1-t) e_{1}\right|^{2}\right)\right)|v|^{2} d x \\
& =\int_{\mathbf{R}^{n}}\left(A \cdot\left(8 \mu^{2}\left(x+R t(1-t) \vec{e}_{1}\right)\right)\right)|v|^{2} d x \\
& =8 \mu^{2} \int_{\mathbf{R}^{n}}\left(A \cdot x+R t(1-t) A^{1}\right)|v|^{2} d x \\
& \geq-8 \mu^{2}\left(C_{1}+\frac{1}{4} R C_{0}\right) \int_{\mathbf{R}^{n}}|v|^{2} d x,
\end{aligned}
$$$$
\text { (by }\|A \cdot x\|_{\infty} \leq C_{1},\|A\|_{\infty} \leq C_{0} \text { ). }
$$

Integration by parts shows that

$$
\begin{aligned}
& -2 \int_{\mathbf{R}^{n}} \sum_{i=1}^{n}\left(\nabla A^{i} \cdot \nabla \partial_{i} v\right) v d x \\
& =2 \int_{\mathbf{R}^{n}}(\Delta A \cdot \nabla v) v d x+2 \int_{\mathbf{R}^{n}}(\nabla A \cdot \nabla v) \cdot \nabla v d x ;
\end{aligned}
$$

we thus have

$$
\begin{aligned}
& -\int_{\mathbf{R}^{n}} \sum_{i=1}^{n}\left(\Delta A^{i} \partial_{i} v\right) v d x-2 \int_{\mathbf{R}^{n}} \sum_{i=1}^{n}\left(\nabla A^{i} \cdot \nabla \partial_{i} v\right) v d x \\
& \quad=\int_{\mathbf{R}^{n}}(\Delta A \cdot \nabla v) v d x+2 \int_{\mathbf{R}^{n}}(\nabla A \cdot \nabla v) \cdot \nabla v d x \\
& \quad \geq-2 C_{0} \int_{\mathbf{R}^{n}}|\nabla v|^{2}-C_{0} \int_{\mathbf{R}^{n}} \frac{1}{2}|\nabla v|^{2} d x-\int_{\mathbf{R}^{n}} 2 C_{0}|v|^{2} d x,
\end{aligned}
$$

(by $\|\nabla A\|_{\infty},\|A\|_{\infty},\|\Delta A\|_{\infty} \leq C_{0}$ and

Cauchy-Schwarz inequality).

Thus, with those inequalities above, we have

$$
\begin{aligned}
\left(\left(\mathcal{S}_{t}\right.\right. & +[\mathcal{S}, \mathscr{A}]) v, v) \\
\geq & \frac{\varepsilon R^{2}}{8 \mu} \int_{\mathbf{R}^{n}}|v|^{2} d x+8 \mu \int_{\mathbf{R}^{n}}|\nabla v|^{2} d x \\
& -\left(2 \mu C_{1}+\frac{1}{2} \mu R C_{0}\right) \int_{\mathbf{R}^{n}}|v|^{2} d x-4 \mu R C_{0} \int_{\mathbf{R}^{n}}|v|^{2} d x \\
& -2 \mu\left(C_{1} C_{0}+C_{0}^{2}+\frac{R}{4} C_{0}^{2}\right) \int_{\mathbf{R}^{n}}|v|^{2} d x \\
& -8 \mu^{2}\left(C_{2}+C_{1}+\frac{R}{4}\left(2 C_{1}+C_{0}\right)+\frac{R^{2}}{4} C_{0}\right) \int_{\mathbf{R}^{n}}|v|^{2} d x \\
& -8 \mu^{2}\left(C_{1}+\frac{1}{4} R C_{0}\right) \int_{\mathbf{R}^{n}}|v|^{2} d x-2 C_{0} \int_{\mathbf{R}^{n}}|\nabla v|^{2} \\
& -C_{0} \int_{\mathbf{R}^{n}} \frac{1}{2}|\nabla v|^{2} d x-\int_{\mathbf{R}^{n}} 2 C_{0}|v|^{2} d x \\
= & \int_{\mathbf{R}^{n}}\left(8 \mu-2 C_{0}-\frac{1}{2} C_{0}\right)|\nabla v|^{2} d x \\
& +\frac{\varepsilon R^{2}}{8 \mu} \int_{\mathbf{R}^{n}}|v|^{2} d x-\int_{\mathbf{R}^{n}} 2 C_{0}|v|^{2} d x-\int_{\mathbf{R}^{n}} 2 \mu^{2} R^{2} C_{0}|v|^{2} d x \\
& -\int_{\mathbf{R}^{n}} 2 \mu\left(C_{1}+C_{1} C_{0}+C_{0}^{2}\right)+8 \mu^{2}\left(C_{2}+2 C_{1}\right)|v|^{2} d x \\
& -\int_{\mathbf{R}^{n}}\left[2 \mu\left(\frac{C_{0}}{4}+2 C_{0}+\frac{C_{0}^{2}}{4}\right) R\right. \\
& \left.+8 \mu^{2}\left(\frac{C_{1}}{2}+\frac{C_{0}}{2}\right) R\right]|v|^{2} d x . \\
&
\end{aligned}
$$

Taking $\varepsilon>(125 / 128) C_{0}^{4}$ and

$$
\sqrt[3]{\frac{\varepsilon}{32 C_{0}}}>\mu>\frac{5 C_{0}}{16},
$$

and letting $R$ be large enough, it follows that

$$
\left(\left(\mathcal{S}_{t}+[\mathcal{S}, \mathscr{A}]\right) v, v\right) \geq \int_{\mathbf{R}^{n}} \frac{\varepsilon R^{2}}{32 \mu}|v|^{2} d x .
$$


Noticing that

$$
\left\|\partial_{t} v-\mathcal{S} v-\mathscr{A} v\right\|_{L^{2}\left(\mathbf{R}^{n} \times[0,1]\right)}^{2} \geq \int_{0}^{1}\left(\left(\mathcal{S}_{t}+[\mathcal{S}, \mathscr{A}]\right) v, v\right) d t,
$$

we have

$$
\left\|\partial_{t} v-\delta v-\mathscr{A} v\right\|_{L^{2}\left(\mathbf{R}^{n} \times[0,1]\right)}^{2} \geq \int_{0}^{1} \int_{\mathbf{R}^{n}} \frac{\varepsilon R^{2}}{32 \mu}|v|^{2} d x d t .
$$

That is

$$
\begin{gathered}
R \sqrt{\frac{\varepsilon}{32 \mu}} \| e^{\mu\left|x+R t(1-t) e_{1}\right|^{2}+\left(\left(R^{2} t(1-t)(1-2 t) / 6\right)-\left((1+\varepsilon) R^{2} t(1-t) / 16 \mu\right)\right)} \\
\quad \times f \|_{L^{2}\left(\mathbf{R}^{n} \times[0,1]\right)} \\
\leq \| e^{\mu\left|x+R t(1-t) e_{1}\right|^{2}+\left(\left(R^{2} t(1-t)(1-2 t) / 6\right)-\left((1+\varepsilon) R^{2} t(1-t) / 16 \mu\right)\right)} \\
\quad \times\left(\partial_{t}-\Delta+A \cdot \nabla\right) f \|_{L^{2}\left(\mathbf{R}^{n} \times[0,1]\right)}
\end{gathered}
$$

This completes the proof of Proposition 7.

The following is to complete the proof of Theorem 1 .

Lemma 8. If $q$ is as in Proposition 5 and satisfies (21), then $q \equiv 0$ in $\mathbf{R}^{n} \times[0,1]$.

Proof. For given $R>0, \gamma>5 C_{0} / 16$ and $\varepsilon>$ $\max \left\{3,(125 / 128) C_{0}^{4}\right\}$; we choose $\mu$ such that

$$
\max \left\{\frac{1}{2}, \frac{5 C_{0}}{16}\right\}<\mu \leq \min \left\{\frac{\gamma}{1+\varepsilon}, \sqrt[3]{\frac{\varepsilon}{64 C_{0}}}\right\} .
$$

Moreover, when $\gamma>5 C_{0} / 16$, with the conditions of Theorem 1 and Proposition 5 and

$$
\left\|e^{14 \gamma|x|^{2}} q(0)\right\|,\left\|e^{14 \gamma|x|^{2}} q(1)\right\|<\infty .
$$

are satisfied, from (88), it follows that

$$
P_{\gamma}=\sup _{[0,1]}\left\|e^{\gamma|x|^{2}} q(t)\right\|+\left\|\sqrt{t(1-t)} e^{\gamma|x|^{2}} \nabla q\right\|_{L^{2}\left(\mathbf{R}^{n} \times[0,1]\right)}<+\infty .
$$

In the Carleman estimate (Proposition 7), let $f(x, t)=$ $\theta_{M}(x) \eta_{R}(t) q(x, t)$, where $\theta_{M}(x), \eta_{R}(t)$ are smooth functions, and $\theta_{M}(x) \in C_{0}^{\infty}\left(\mathbf{R}^{n}\right)$ verifies

$$
\begin{array}{ll}
\theta_{M}(x)=1, & |x| \leq M, \\
\theta_{M}(x)=0, & |x| \geq 2 M,
\end{array}
$$

where $M \geq R$ and $\eta_{R}(t) \in C_{0}^{\infty}(0,1)$ verifies

$$
\begin{gathered}
\eta_{R}(t)=1, \quad t \in\left[\frac{1}{R}, 1-\frac{1}{R}\right], \\
\eta_{R}(t)=0, \quad t \in\left[0, \frac{1}{2 R}\right] \cup\left[1-\frac{1}{2 R}, 1\right] .
\end{gathered}
$$

Then, in $\mathbf{R}^{n} \times[0,1], f$ is compact supported.
A calculation shows that

$$
\begin{aligned}
\partial_{t} f-\Delta & f+A \cdot \nabla f+b(f) \\
= & \theta_{M} \eta_{R}^{\prime} q+\theta_{M} \eta_{R} \partial_{t} q \\
& -\left(\theta_{M} \eta_{R} \Delta q+2 \eta_{R} \nabla \theta_{M} \cdot \nabla q+\eta_{R} q \Delta \theta_{M}\right) \\
& +\theta_{M} \eta_{R} A \cdot \nabla q+\eta_{R} q A \cdot \nabla \theta_{M}+b\left(\theta_{M} \eta_{R} q\right) \\
= & \theta_{M} \eta_{R}\left(\partial_{t} q-\Delta q+A \cdot \nabla q+B q\right) \\
& +\theta_{M} \eta_{R}{ }^{\prime} q-\left(2 \nabla \theta_{M} \cdot \nabla q+q \Delta \theta_{M}+q A \cdot \nabla \theta_{M}\right) \eta_{R} .
\end{aligned}
$$

From

$$
\partial_{t} q-\Delta q+A \cdot \nabla q+B q=0
$$

it follows that

$$
\begin{aligned}
\partial_{t} f & -\Delta f+A \cdot \nabla f+b(f) \\
& =\theta_{M} \eta_{R}{ }^{\prime} q-\left(2 \nabla \theta_{M} \cdot \nabla q+q \Delta \theta_{M}+q A \cdot \nabla \theta_{M}\right) \eta_{R},
\end{aligned}
$$

where $\theta_{M} \eta_{R}{ }^{\prime} q$ is supported in $B_{2 M} \times[1 / 2 R, 1 / R] \cup[1-1 / R, 1-$ $1 / 2 R]$, and, from Hölder inequality and the range of $t$, we have

$$
\begin{aligned}
\mu \mid x & +\left.R t(1-t) e_{1}\right|^{2} \\
& =\mu|x|^{2}+2 \mu R t(1-t) x_{1}+\mu[R t(1-t)]^{2} \\
& \leq \mu|x|^{2}+\mu\left(\varepsilon|x|^{2}+\frac{[R t(1-t)]^{2}}{\varepsilon}\right)+\mu[R t(1-t)]^{2} \\
& =\mu(1+\varepsilon)|x|^{2}+\mu\left(1+\frac{1}{\varepsilon}\right)[R t(1-t)]^{2} \\
& \leq \mu(1+\varepsilon)|x|^{2}+\mu\left(1+\frac{1}{\varepsilon}\right) .
\end{aligned}
$$

Noticing that $\mu \leq \gamma /(1+\varepsilon)$, we have

$$
\mu\left|x+R t(1-t) \vec{e}_{1}\right|^{2} \leq \gamma|x|^{2}+\frac{\gamma}{\varepsilon} .
$$

In the same way, $-\left(2 \nabla \theta_{M} \cdot \nabla q+q \Delta \theta_{M}+q A \cdot \nabla \theta_{M}\right) \eta_{R}$ of (126) is supported in $B_{2 M} \backslash B_{M} \times[1 / 2 R, 1-1 / 2 R]$, and

$$
\begin{aligned}
& \mu\left|x+R t(1-t) e_{1}\right|^{2} \\
& \quad \leq \mu(1+\varepsilon)|x|^{2}+\mu\left(1+\frac{1}{\varepsilon}\right)[R t(1-t)]^{2} \\
& \quad \leq \gamma|x|^{2}+\frac{\gamma R^{2}}{\varepsilon} .
\end{aligned}
$$


Hence, in each of the parts of the support of $\partial_{t} f-\Delta f+A$. $\nabla f+b(f), \mu\left|x+R t(1-t) \vec{e}_{1}\right|^{2}$ are bounded, and applying Proposition 7 to $f$, we get

$$
\begin{aligned}
& R \sqrt{\frac{\varepsilon}{32 \mu}}\left\|e^{\varphi} f\right\|_{L^{2}\left(\mathbf{R}^{n} \times[0,1]\right)} \\
& \leq\left\|e^{\varphi}\left(\partial_{t}-\Delta+A \cdot \nabla\right) f\right\|_{L^{2}\left(\mathbf{R}^{n} \times[0,1]\right)} \\
& =\| e^{\varphi}\left[(-B f)+\theta_{M} \eta_{R}^{\prime} q\right. \\
& \left.\quad-\left(2 \nabla \theta_{M} \cdot \nabla q+q \Delta \theta_{M}+q A \cdot \nabla \theta_{M}\right) \eta_{R}\right] \|_{L^{2}\left(\mathbf{R}^{n} \times[0,1]\right)}
\end{aligned}
$$

The natural bounds for $\theta_{M}, \eta_{R}, \nabla \theta_{M}$, and $\Delta \theta_{M}$ show that there is a constant $P_{\varepsilon}$ such that

$$
\begin{aligned}
& R\left\|e^{\varphi} f\right\|_{L^{2}\left(\mathbf{R}^{n} \times[0,1]\right)} \\
& \leq P_{\varepsilon}\|B\|_{L^{\infty}\left(\mathbf{R}^{n} \times[0,1]\right)}\left\|e^{\varphi} f\right\|_{L^{2}\left(\mathbf{R}^{n} \times[0,1]\right)}+P_{\varepsilon} R \sup _{[0,1]}\left\|e^{\varphi} q\right\| \\
& \quad+P_{\varepsilon} M^{-1}\left\|e^{\varphi}(|\nabla q|+|q|)\right\|_{L^{2}\left(\mathbf{R}^{n} \times[1 / 2 R, 1-1 / 2 R]\right)} .
\end{aligned}
$$

From (128), (129), and (131), it follows that

$$
\begin{aligned}
R\left\|e^{\varphi} f\right\|_{L^{2}\left(\mathbf{R}^{n} \times[0,1]\right)} & \\
\leq & P_{\varepsilon}\|B\|_{L^{\infty}\left(\mathbf{R}^{n} \times[0,1]\right)}\left\|e^{\varphi} f\right\|_{L^{2}\left(\mathbf{R}^{n} \times[0,1]\right)}+P_{\varepsilon} R e^{\gamma / \varepsilon} \sup _{[0,1]}\left\|e^{\gamma|x|^{2}} q\right\| \\
& +P_{\varepsilon} M^{-1} e^{\gamma R^{2} / \varepsilon}\left\|e^{\gamma|x|^{2}}(|\nabla q|+|q|)\right\|_{L^{2}\left(\mathbf{R}^{n} \times[1 / 2 R, 1-1 / 2 R]\right)} .
\end{aligned}
$$

When $P_{\varepsilon}\|B\|_{L^{\infty}\left(R^{n} \times[0,1]\right)} \leq R / 2$ and $M \rightarrow \infty$, the last tends to zero by (121). Therefore

$$
\frac{R}{2}\left\|e^{\varphi} f\right\|_{L^{2}\left(\mathbf{R}^{n} \times[0,1]\right)} \leq P_{\varepsilon} R e^{\gamma / \varepsilon} \sup _{[0,1]}\left\|e^{\gamma|x|^{2}} q\right\| .
$$

$$
\begin{aligned}
\text { For }|x| & \leq \varepsilon R / 4, \mu>1 / 2, \varepsilon>3 \\
\varphi\left(x, \frac{1}{2}\right) & =\mu\left|x+\frac{R e_{1}}{4}\right|^{2}-\frac{(1+\varepsilon) R^{2}}{64 \mu} \\
& \geq \mu\left|-\frac{\varepsilon R}{4}+\frac{R}{4}\right|^{2}-\frac{(1+\varepsilon) R^{2}}{64 \mu} \\
& =\frac{R^{2}}{64 \mu}\left(4 \mu^{2}(1-\varepsilon)^{2}-(1+\varepsilon)\right)=K(\gamma, \varepsilon) R^{2}>0
\end{aligned}
$$

while $\varphi$ is continuous about $t$, so there is $\varepsilon_{1}>0$, in $B_{(\varepsilon R / 4) \times\left[\left(1-\varepsilon_{1}\right) / 2,\left(1+\varepsilon_{1}\right) / 2\right]}$,

$$
\varphi(x, t) \geq \frac{K(\gamma, \varepsilon) R^{2}}{2}>0 .
$$

This inequality, the fact $f=q$ in $B_{(\varepsilon R / 4) \times\left[\left(1-\varepsilon_{1}\right) / 2,\left(1+\varepsilon_{1}\right) / 2\right]}$ with the value ranges of $\mu$, and (133) together show that

$$
\frac{R}{2} e^{K(\gamma, \varepsilon) R^{2} / 2}\|q\|_{L^{2}\left(B_{(\varepsilon R / 4) \times\left[\left(1-\varepsilon_{1}\right) / 2,\left(1+\varepsilon_{1}\right) / 2\right]}\right)} \leq P_{\gamma, \varepsilon} R,
$$

when $P_{\varepsilon}\|B\|_{L^{\infty}\left(\mathbf{R}^{n} \times[0,1]\right)} \leq R / 2$.

Thus, when $R \rightarrow \infty,\|q\|_{L^{2}\left(B_{(\varepsilon R / 4) \times\left[\left(1-\varepsilon_{1}\right) / 2,\left(1+\varepsilon_{1}\right) / 2\right]}\right)}=0$. And then, when $t \in\left[\left(1-\varepsilon_{1}\right) / 2,\left(1+\varepsilon_{1}\right) / 2\right]$, and $\varepsilon>3,\|q\|_{L^{2}\left(B_{\varepsilon R / 4}\right)}=$ 0 .

On the other hand, when $t \in\left[\left(1-\varepsilon_{1}\right) / 2,\left(1+\varepsilon_{1}\right) / 2\right]$,

$$
\begin{aligned}
& \|q(t)\|_{L^{2}\left(R^{n}\right)} \\
& \quad \leq\|q(t)\|_{L^{2}\left(B_{\varepsilon R / 4}\right)}+\|q(t)\|_{L^{2}(|x|>\varepsilon R / 4)} \\
& \leq\|q(t)\|_{L^{2}\left(B_{\varepsilon R / 4}\right)}+\sup _{[0,1]}\|q(t)\|_{L^{2}(|x|>\varepsilon R / 4)} .
\end{aligned}
$$

From (121)

$$
\sup _{[0,1]}\|q(t)\|_{L^{2}(|x|>\varepsilon R / 4)} \leq e^{-\varepsilon^{2} R^{2} / 16} P_{\gamma} .
$$

So, from (137), we have

$$
\|q(t)\|_{L^{2}\left(\mathbf{R}^{n}\right)} \leq\|q(t)\|_{L^{2}\left(B_{\varepsilon R / 4}\right)}+e^{-\varepsilon^{2} R^{2} / 16} P_{\gamma} .
$$

Thus, when $R \rightarrow \infty$,

$$
\|q(t)\|_{L^{2}\left(\mathbf{R}^{n}\right)}=0, \quad t \in\left[\frac{1-\varepsilon_{1}}{2}, \frac{1+\varepsilon_{1}}{2}\right] .
$$

This means that $q=0$ when $(x, t) \in \mathbf{R}^{n} \times\left[\left(1-\varepsilon_{1}\right) / 2,\left(1+\varepsilon_{1}\right) / 2\right]$. With the standard uniqueness and the backward uniqueness theorem for parabolic equations (see $[18,20]$ for detail), $q \equiv 0$, $(x, t) \in \mathbf{R}^{n} \times[0,1]$. This completes the proof of Lemma 8 .

From Lemma 8, we have the following corollary.

Corollary 9. If $u \in C^{1}\left(\mathbf{R}^{n} \times[0,1]\right)$, then $u \equiv 0$.

Proof. If $u \in C^{1}\left(\mathbf{R}^{n} \times[0,1]\right)$, from Lemma 8 , we can get

$$
q \equiv 0 \quad \text { in } \mathbf{R}^{n} \times[0,1]
$$

that is, $\operatorname{curl} u(\cdot, t) \equiv 0$. Hence, there is a function $v \in C^{2}\left(\mathbf{R}^{n} \times\right.$ $[0,1])$, and $\nabla_{x} v=u$.

Form $\nabla \cdot u(\cdot, t)=0$, we have

$$
\Delta_{x} v=0
$$

For (142), there is a solution $v \equiv C, v \in C^{2}\left(\mathbf{R}^{n} \times[0,1]\right)$, with

$$
\|u\|_{L^{\infty}\left(\mathbf{R}^{n} \times[0,1]\right)} \leq C_{0}, \quad\|u \cdot x\|_{L^{\infty}\left(\mathbf{R}^{n} \times[0,1]\right)} \leq C_{2} .
$$

So, $u=\nabla v \equiv 0$, when $u \in C^{1}\left(\mathbf{R}^{n} \times[0,1]\right)$. This completes the proof of Theorem 1 .

\section{Conflict of Interests}

The authors declare that there is no conflict of interests regarding the publication of this paper. 


\section{Acknowledgment}

This paper is funded by National Natural Science Foundation of China (no. 61272033).

\section{References}

[1] J. Leray, "Sur le mouvement d'un liquide visqueux emplissant l'espace," Acta Mathematica, vol. 63, no. 1, pp. 193-248, 1934.

[2] O. A. Ladyzhenskaya, Mathematical Problems of the Dynamics of Viscous Incompressible Fluids, Nauka, Moscow, Russia, 2nd edition, 1970.

[3] P. L. Lions, Mathematical Topics in Fluid Mechanics: Volume 1: Incompressible Models, Oxford University Press, New York, NY, USA, 1996.

[4] R. Temam, Navier-Stokes Equations, North-Holland, Amsterdam, The Netherlands, 1984.

[5] B. Q. Dong and Z. Zhang, "On the weak-strong uniqueness of Koch-Tataru's solution for the Navier-Stokes equations," Journal of Differential Equations, vol. 256, no. 7, pp. 2406-2422, 2014.

[6] M. Cannone, F. Planchon, and M. Schonbek, "Strong solutions to the incompressible Navier-Stokes equations in the halfspace," Communications in Partial Differential Equations, vol. 25, no. 5-6, pp. 903-924, 2000.

[7] H. Koch and D. Tataru, "Well-posedness for the Navier-Stokes equations," Advances in Mathematics, vol. 157, no. 1, pp. 22-35, 2001.

[8] O. A. Ladyzhenskaya, "Uniqueness and smoothness of generalized solutions of Navier-Stokes equations," Zapiski Nauchnyh Seminarov POMI, vol. 5, pp. 169-185, 1967.

[9] Z. Lei and F. Lin, "Global mild solutions of Navier-Stokes equations," Communications on Pure and Applied Mathematics, vol. 64, no. 9, pp. 1297-1304, 2011.

[10] A. Biswas, "Gevrey regularity for a class of dissipative equations with applications to decay," Journal of Differential Equations, vol. 253, no. 10, pp. 2739-2764, 2012.

[11] L. Caffarelli, R. Kohn, and L. Nirenberg, "Partial regularity of suitable weak solutions of the Navier-Stokes equations," Communications on Pure and Applied Mathematics, vol. 35, no. 6, pp. 771-831, 1982.

[12] M. Struwe, "On partial regularity results for the Navier-Stokes equations," Communications on Pure and Applied Mathematics, vol. 41, no. 4, pp. 437-458, 1988.

[13] C. Amrouche, V. Girault, M. E. Schonbek, and T. P. Schonbek, "Pointwise decay of solutions and of higher derivatives to Navier-Stokes equations," SIAM Journal on Mathematical Analysis, vol. 31, no. 4, pp. 740-753, 2000.

[14] W. Borchers and T. Miyakawa, "Algebraic $L_{2}$ decay for NavierStokes flows in exterior domains," Acta Mathematica, vol. 165, no. 3-4, pp. 189-227, 1990.

[15] W. Borchers and T. Miyakawa, " $L_{2}$ decay for the Navier-Stokes flow in halfspaces," Mathematische Annalen, vol. 282, no. 1, pp. 139-155, 1988.

[16] T. Miyakawa and H. Sohr, "On energy inequality, smoothness and large time behavior in $L^{2}$ for weak solutions of the Navier-Stokes equations in exterior domains," Mathematische Zeitschrift, vol. 199, no. 4, pp. 455-478, 1988.

[17] M. E. Schonbek, " $\mathrm{L}^{2}$ decay for weak solutions of the NavierStokes equations," Archive for Rational Mechanics and Analysis, vol. 88 , no. 3, pp. 209-222, 1985.
[18] L. Escauriaza, G. Seregin, and V. Šverák, "Backward uniqueness for parabolic equations," Archive for Rational Mechanics and Analysis, vol. 169, no. 2, pp. 147-157, 2003.

[19] G. P. Galdi and B. Straughan, "Stability of solutions of the Navier-Stokes equations backward in time," Archive for Rational Mechanics and Analysis, vol. 101, no. 2, pp. 107-114, 1988.

[20] D. Hoff and E. Tsyganov, "Time analyticity and backward uniqueness of weak solutions of the Navier-Stokes equations of multidimensional compressible flow," Journal of Differential Equations, vol. 245, no. 10, pp. 3068-3094, 2008.

[21] L. Hörmander, The Analysis of Linear Partial Differential Operators III, IV, Springer, Berlin, Germany, 1990.

[22] N. Garofalo and Z. Shen, "Carleman estimates for a subelliptic operator and unique continuation," Annales de l'Institut Fourier, vol. 44, no. 1, pp. 129-166, 1994.

[23] J. Saut and B. Scheurer, "Unique continuation for some evolution equations," Journal of Differential Equations, vol. 66, no. 1, pp. 118-139, 1987.

[24] X. Chen, "A strong unique continuation theorem for parabolic equations," Mathematische Annalen, vol. 311, no. 4, pp. 603-630, 1998.

[25] R. Regbaoui, "Strong unique continuation for Stokes equations," Communications in Partial Differential Equations, vol. 24, no. 910, pp. 1891-1902, 1999.

[26] M. Ignatova and I. Kukavica, "Strong unique continuation for the Navier-Stokes equation with non-analytic forcing," Journal of Dynamics and Differential Equations, vol. 25, no. 1, pp. 1-15, 2013.

[27] K. Masuda, "On the analyticity and the unique continuation theorem for solutions of the Navier-Stokes equation," Proceedings of the Japan Academy, vol. 43, pp. 827-832, 1967.

[28] A. D. Ionescu and C. E. Kenig, " $L$-Carleman inequalities and uniqueness of solutions of nonlinear Schrödinger equations," Acta Mathematica, vol. 193, no. 2, pp. 193-239, 2004.

[29] Z. Duan and P. Li, "On unique continuation for the generalized Schrödinger equations," Journal of Mathematical Analysis and Applications, vol. 420, no. 2, pp. 1719-1743, 2014.

[30] J. Bourgain, "On the compactness of the support of solutions of dispersive equations," International Mathematics Research Notices, vol. 9, pp. 437-447, 1997.

[31] L. Escauriaza, C. E. Kenig, G. Ponce, and L. Vega, "Hardy's uncertainty principle, convexity and Schrödinger evolutions," Journal of the European Mathematical Society (JEMS), vol. 10, no. 4, pp. 883-907, 2008.

[32] C. E. Kenig, G. Ponce, and L. Vega, "On unique continuation for nonlinear Schrödinger equations," Communications on Pure and Applied Mathematics, vol. 56, no. 9, pp. 1247-1262, 2003.

[33] R. Finn, "Stationary solutions of the Navier-Stokes equations," Proceedings of Symposia in Applied Mathematics: American Mathematical Society, vol. 17, pp. 121-153, 1965.

[34] R. H. Dyer and D. E. Edmunds, "Asymptotic behaviour of solutions of the stationary Navier-Stokes equations," Journal of the London Mathematical Society. Second Series, vol. 44, pp. 340-346, 1969.

[35] G. P. Galdi, An Introduction to the Mathematical Theory of the Navier-Stokes Equations, I and II, Springer, New York, NY, USA, 1994.

[36] C. Lin, G. Uhlmann, and J. Wang, "Optimal three-ball inequalities and quantitative uniqueness for the Stokes system," Discrete and Continuous Dynamical Systems A, vol. 28, no. 3, pp. 12731290, 2010. 
[37] C. L. Lin, G. Uhlmann, and J. N. Wang, "Asymptotic behavior of solutions of the stationary Navier-Stokes equations in an exterior domain," http://arxiv.org/abs/1008.3953.

[38] C.-L. Lin, G. Nakamura, and J.-N. Wang, "Optimal three-ball inequalities and quantitative uniqueness for the Lamé system with Lipschitz coefficients," Duke Mathematical Journal, vol. 155, no. 1, pp. 189-204, 2010.

[39] A. Bonami and B. Demange, "A survey on uncertainty principles related to quadratic forms," Collectanea Mathematica, pp. 1-36, 2006.

[40] L. C. Evans, Partial Differential Equations, American Mathematical Society, 1998. 


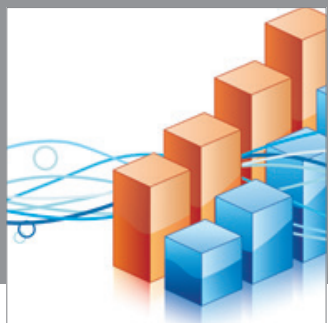

Advances in

Operations Research

mansans

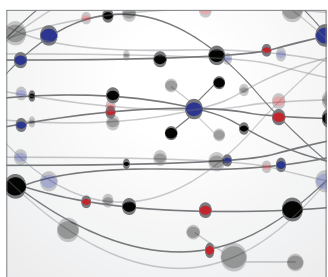

The Scientific World Journal
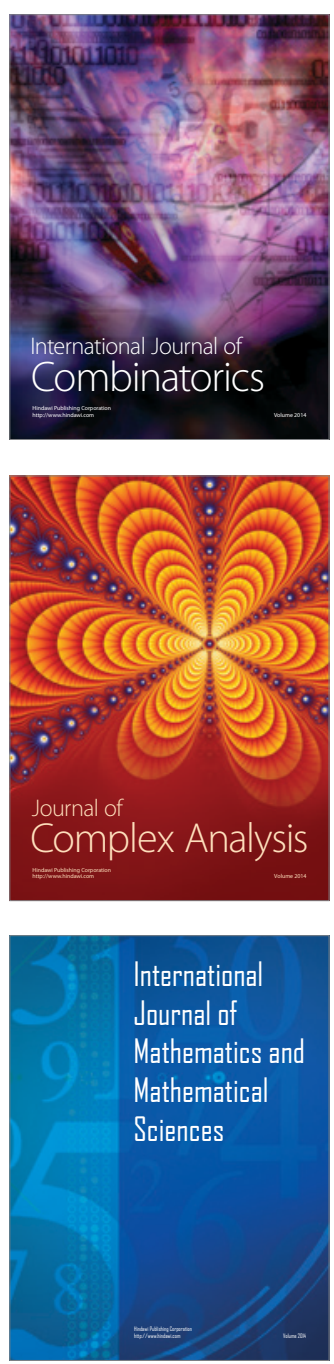
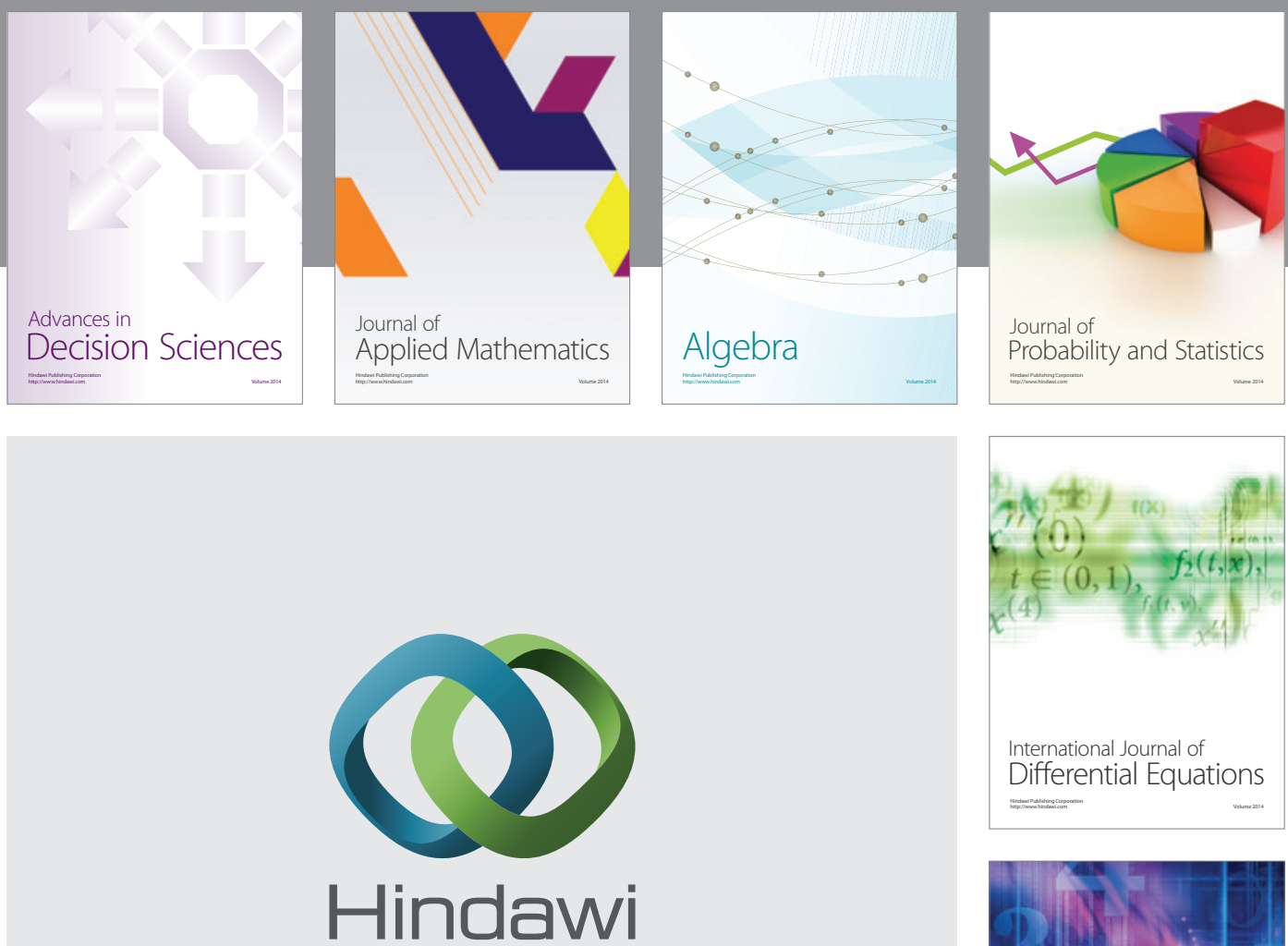

Submit your manuscripts at http://www.hindawi.com
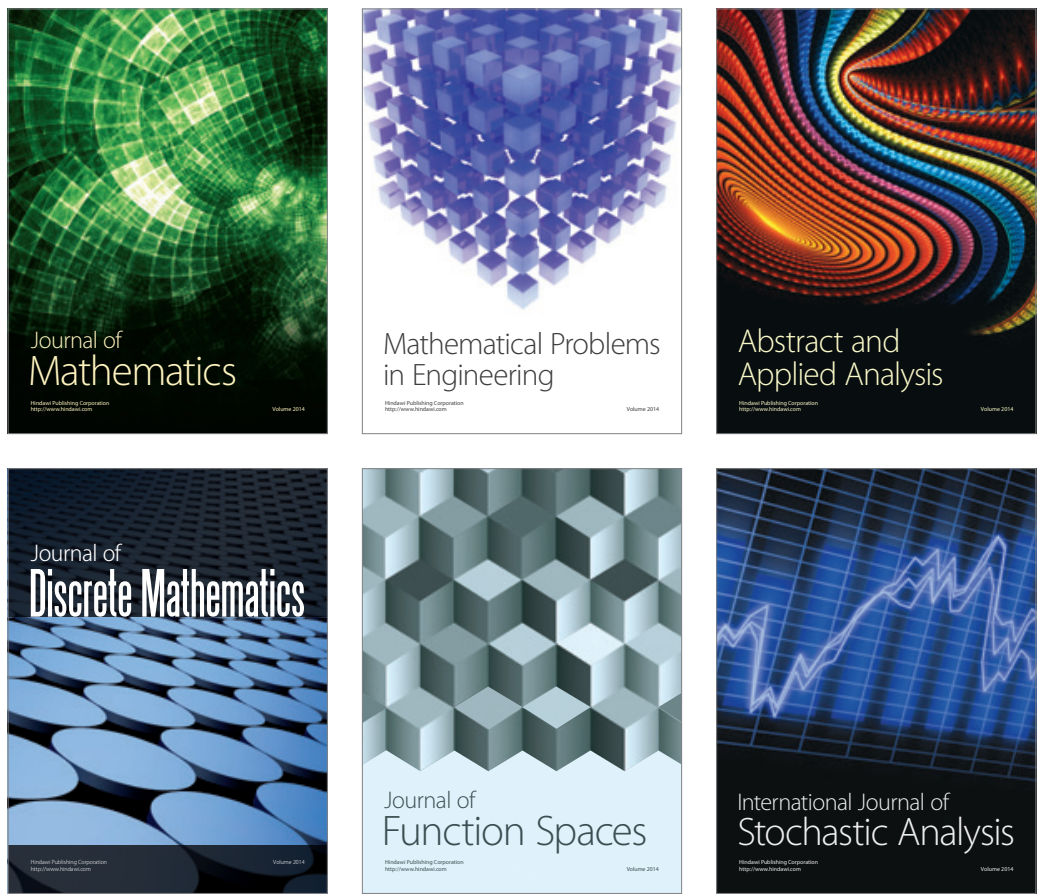

Journal of

Function Spaces

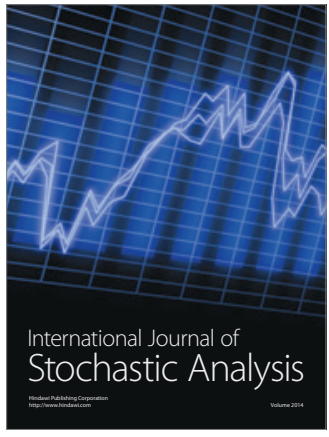

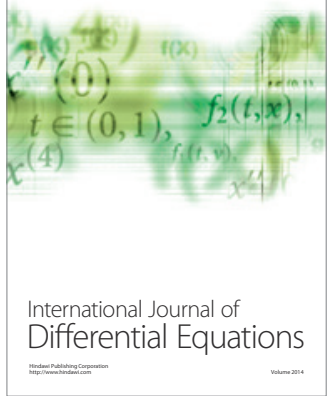
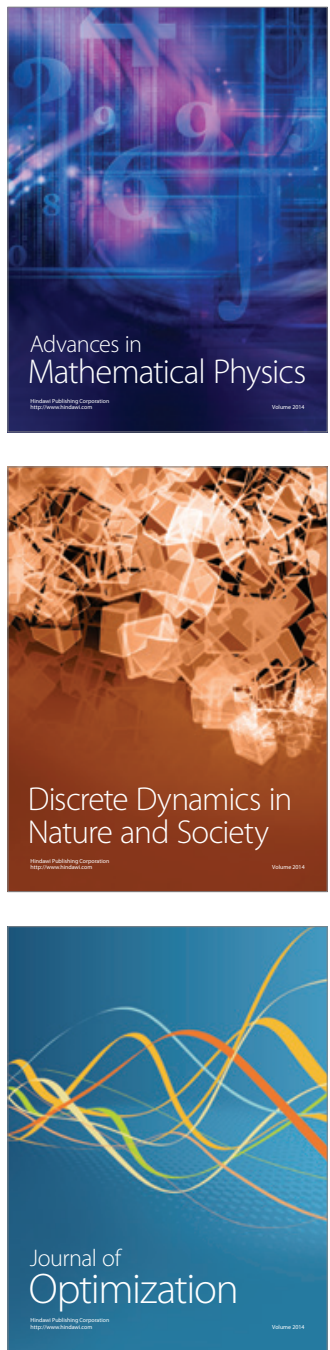